SAE 88-1448

\title{
LDV Surveys Over a Fighter Model at Moderate to High Angles of Attack
}

William L. Sellers III, James F. Meyers NASA Langley Research Center Hampton, VA

Timothy E. Hepner

United States Army (AVRADCOM) Hampton, VA

SAE AEROTECH 88

October 3-6, 1988

Anaheim, California 



\title{
LDV Surveys Over a Fighter Model at Moderate to High Angles of Attack
}

\author{
William L. Sellers III \\ James F. Meyers \\ NASA Langley Research Center \\ Hampton, VA \\ Timothy E. Hepner \\ United States Army (AVRADCOM) \\ Hampton, VA
}

\begin{abstract}
The vortex flowfield over an advanced twin-tailed fighter configuration was measured in a low-speed wind tunnel at two angles of attack. The primary test data consisted of 3-component velocity surveys obtained using a Laser Doppler Velocimeter. Laser light sheet and surface flow visualization were also obtained to provide insight into the flowfield structure. Time-averaged velocities and the root mean square of the velocity fluctuations were obtained at two cross-sections above the model. At 15 degrees angle of attack, the vortices generated by the wing leading edge extension (LEX) were unburst over the model and passed outboard of the vertical tail. At 25 degrees angle of attack, the vortices burst in the vicinity of the wing-LEX intersection and impact directly on the vertical tails. The RMS levels of the velocity fluctuations reach values of approximately $30 \%$ in the region of the vertical tails.
\end{abstract}

MODERN FIGHTER AIRCRAFT at high angles of attack generate vortices which produce substantial increases in lift. The vortices are shed by the sharp wing leading edges, slender fuselage forebodies, and by the highly-swept wing leading-edge extensions (LEXs). There is also interest in techniques which exploit the vortices to provide increases in maneuverability in the high-alpha regime where conventional control surfaces lose their effectiveness (1).* Figure 1 is a photograph of an F-18 aircraft during a high angle of attack maneuver and shows the LEX vortices which are made visible by natural condensation of water vapor.

* Numbers in parentheses designate references at end of paper. 
The interaction of the vortices with the tail surfaces can cause structural problems. Both the F-15 (2) and the F-18 (3), have encountered buffeting and/or structural fatigue problems with the vertical tails at high angles-of-attack.

The buffeting is due in a large part to the breakdown of the vortices ahead of the tails. Vortex bursting or breakdown is meant to describe the condition where the axial velocity at the core of the vortex abruptly stagnates followed by a rapid expansion of the vortex core. After the expansion the flow changes to a highly-turbulent swirling state.

The lack of quantitative flowfield data has hampered efforts to develop theoretical methods to predict aircraft buffet or a buffet design criteria for the high angle of attack flight regime. There have been numerous research papers written on the subject of vortex flows from slender delta wings, but much of this data is of limited use, since the instrumentation that was available at the time was not capable of measuring the velocity field in a burst vortex. Flowfield data above configurations are even more scarce. The existing data base consists mostly of force and moment measurements, surface pressures, and surface or off-body flow visualizations. A great deal of data were obtained at low Reynolds number in water tunnels and these data provide a valuable insight into the vortex interactions. Quantitative spectral data was obtained by Wentz (3) using a hot-film sensor mounted on the vertical tail of an F-18 model in a water tunnel. Wentz found that dominant frequencies appear in the flow when bursting occurs and that these frequencies correspond to a Strouhal number of about 0.7 for the range of speeds that were tested.

Velocity data and quantitative measurements of the vortex strength and characteristics will be required before computational codes can be validated or empirical methods developed. Lan reviewed various methods of predicting aircraft buffeting in (4). He developed an analytical method to generate a fluctuating flowfield, which then produces unsteady pressures on the vertical tail of the F-18. This method is based on test data of the mean square values of the fluctuating normal force. Lan suggests that there is a strong need for the measurement of the fluctuating velocity components in a buffeting flowfield.

This investigation represents a small part of a broad NASA wide effort to improve the fundamental understanding of aerodynamic flows at high angle of attack. The purpose was to obtain detail flowfield measurements in the vortex dominated flowfield around the YF- 17 fighter configuration. It was a cooperative effort between the NASA Langley Research Center and the Aircraft Division of the Northrop 
Corporation. A three component Laser Doppler Velocimeter (LDV) was used to measure the mean velocity and the RMS of the fluctuating velocity components above the model. The Northrop YF-17 configuration was the prototype for the McDonnell Douglas F/A-18 Hornet. The YF-17 shown in the three-view sketch in figure 2, incorporates design features that are typical of many of the current generation fighter aircraft. It includes a moderately-swept $\left(26.6^{\circ}\right)$ wing, twin vertical tails, and a highly-swept $\left(80^{\circ}\right)$ leading edge extension. The flowfield generated by this aircraft exhibits many of the characteristics of the present generation fighter aircraft, as well as the problems such as vortex interaction with the tail surfaces.

\section{TEST FACILITY}

The test was conducted in the NASA Langley Basic Aerodynamics Research Tunnel (BART). The facility was developed to acquire the detailed flowfield data required for computational code validation. The facility is ideally suited for flowfield surveys over complex aircraft configurations. The following sections will briefly describe the facility and data systems that were primarily used during this investigation. A detailed description of the tunnel is provided in (5) along with numerous examples demonstrating the various instrumentation systems and flow survey capabilities.

Basic Aerodynamics Research Tunnel The tunnel shown in figure 3 , is an open-return wind tunnel with a test section 28 inches high, 40 inches wide and 10 feet long. The test section is divided into 2 -five foot long bays. The maximum flow velocity in the test section is $220 \mathrm{ft} / \mathrm{sec}$ which yields a $R_{n} / \mathrm{ft}$ of 1.4 million. The airflow entering the test section is conditioned by a honeycomb, 4 anti-turbulence screens and an 11:1 contraction ratio. The four-inch thick honeycomb has a 0.25 inch cell size. The screens are 20 meshes per inch with a porosity (ratio of open area to closed area) of $64 \%$. The flow conditioners provide a low-turbulence, uniform flow in the test section in which the u-component of the turbulence intensity ranges from $.05 \%$ at $q=10 \mathrm{lb} / \mathrm{ft}^{2}(V=92 \mathrm{ft} / \mathrm{sec})$ to $.08 \%$ at $q=45 \mathrm{lb} / \mathrm{ft}^{2}(V=195 \mathrm{ft} / \mathrm{sec})$.

\section{Data Acquisition And Control System The BART tunnel is} equipped with a highly integrated and automated data acquisition and control system (DACS). The system incorporates real-time color graphic displays of flowfield parameters which are required to ensure that the experiment is progressing properly and to assimilate the large amount of information that is acquired. 
The DACS is an integration of two different computer systems. The Tunnel Data Acquisition and Control (TDAC) computer system is used to acquire low transfer rate data from the tunnel and several peripheral systems. The TDAC also drives a 2-axis traverse system which is used for pressure probe surveys or for the LDV seeding system. Data acquisition software has been written which allows completely automated surveys of flowfields above models of arbitrary geometric shape. The TDAC provides the real-time color graphic display of the flowfield parameters to monitor the progress of the test. A larger minicomputer, which will be described later, handles the on-line acquisition and reduction of the 3 component LDV data and postprocessing of the flowfield data.

Laser Doppler Velocimeter System BART is equipped with a dedicated, 3-component LDV system to enable the non-intrusive measurement of flowfields. The LDV is one of the few instruments capable of obtaining velocity measurements in flowfields with reverse flows, large shear gradients and velocity fluctuations.

The LDV system is an orthogonal crossed-fringe configuration with the receive optics mounted $90^{\circ}$ off-axis. The 514.5, 496.5, and 476.5 nanometer wavelengths are used to measure the lateral $(v)$, streamwise $(u)$, and vertical $(w)$ velocity components respectively. Bragg cells are used to provide directional measurement capability in all three velocity components. The sample volume is spherical in shape and has been calculated to be approximately $150-\mu \mathrm{m}$ in diameter. The optics and laser move as a unit on a traversing system that provides 1 meter of travel, with $10-\mu \mathrm{m}$ resolution in all three axes. A detailed description of the design of the LDV system is provided in (6). Figure 4 is a photograph showing the installation of the traversing system around the test section of the tunnel. Figure 5 is a photograph of the laser beams crossing over the YF-17 model. The $u$ and $w$ components are measured using the four horizontal beams and the $v$ component is measured using the two vertical beams. The sample volume is located at the intersection of the six beams.

The flowfield is seeded with monodisperse $0.8-\mu \mathrm{m}$ polystyrene latex microspheres. The seed particles are suspended in a mixture of alcohol and water and are injected into the flow upstream of the honeycomb using an atomizing spray nozzle. The particles are fabricated at NASA Langley using the technique described by Nichols in (7).

The ability of a particle to track the streamlines in the flowfield, and thus the ultimate accuracy of the LDV, is related to the size of the particle. Theoretical predictions of particle trajectories in swirling flows were reported by Dring and Suo (8). They concluded that the 
particle trajectory in a free vortex swirling flow is governed by the Stokes number $\left(S_{t}\right)$ and when the Stokes number is less than 0.1 , the particle will follow the circular streamlines of a free vortex. The $0.8-\mu \mathrm{m}$ particles used in this test, have a density $\rho_{p}=2.03727 \mathrm{slugs} / \mathrm{ft}^{3}$. These particles were shown in (5) to be able to follow the streamlines of the vortices generated by a $75^{\circ}$ delta wing at $20.5^{\circ}$ angle of attack using the numerical procedure described by Dring.

The LDV data acquisition system is highly automated and fully integrated into the tunnel data system as described earlier. The LDV system can obtain velocity measurements in either an automated, step or manual mode. In the automated mode of operation, the system can survey an entire cross flow plane in a completely automated fashion, based on a previously generated file of grid coordinates. In the step mode of operation, the position of the sample volume is still read from the grid file, but the system pauses at the new location until an acquire data command is input from the terminal. This mode is typically used when measuring velocities in difficult regions where the instrumentation must be checked to verify the processing of valid signals.

The signals from the photodetectors are input to high-speed burst counters where the frequency of the light scattered from the particles is determined and the signals verified. The data are then transferred to the laser velocimeter autocovariance buffer interface (LVABI) for temporary storage during the acquisition cycle (9). Each velocity channel has a companion interarrival time channel that measures the time between successive velocity measurements. The LVABI provides a maximum of $64 \mathrm{k}$ words of storage per channel. The actual buffer size is programmable in blocks from $1 \mathrm{~K}$ to $64 \mathrm{~K}$ and for this test it was set to 4096. When the desired number of measurements is acquired or the acquisition time (typically one minute) has elapsed, the acquired velocity and interarrival time data for each velocity component are transferred to the host minicomputer for on-line processing and storage.

The host minicomputer computes the statistical moments of the velocity samples at each measurement location and displays on-line quantities such as the velocity mean, standard deviation, skew and excess. The standard deviation $(\sigma)$ is used as a measure of the RMS velocity fluctuations and is typically non-dimensionalized by the freestream velocity to provide a measure of the relative turbulence intensity. The mean and $\sigma$ for each velocity component are passed to the TDAC computer for input to a color graphic display of the flowfield. 


\section{MODEL DESCRIPTION}

The model used in this investigation was a $3 \%$ scale model of the Northrop YF-17. The model had an overall fuselage length of 18.9 inches, a wing span of 12.6 inches, and a mean aerodynamic chord (MAC) of 3.88 inches. The YF-17 aircraft incorporates a programmed wing leadingedge flap which deflects as a function of angle of attack. The model used in this test, however, was not equipped with leading edge flaps. In addition, the model was tested without the wing tip missiles and launcher rails. The model was attached to a small sting that was mounted on the BART model support system. A photograph of the model installed in the test section of the tunnel is shown in figure 6 .

\section{TEST CONDITIONS}

All data were obtained at a free stream dynamic pressure of either 10 or $30 \mathrm{lb} / \mathrm{ft}^{2}$, which represents free stream velocities of 92 or $159 \mathrm{ft} / \mathrm{sec}$, respectively. These two speeds resulted in test Reynolds numbers of 188,000 or 326,000 based on the MAC. The two angles of attack ( $\alpha=15^{\circ}$ and $25^{\circ}$ ) investigated represented conditions where the vortices over the model would be unburst and burst, respectively. The higher angle of attack represented a condition where, based on Northrop YF-17 flight test data, the tail buffet loads increase significantly. The two survey stations (Sta. 440 and 524) where flowfield data were obtained are shown in figure 2. Velocity measurements were acquired over the right half of the model. This paper will only present the data acquired at station 524 for the $R_{n}=326,000$ condition.

\section{FLOW VISUALIZATION}

Flow visualization was used to obtain a rapid global look at the flowfield and to determine areas of interest for later detailed investigation. A titanium dioxide $\left(\mathrm{TiO}_{2}\right)$ surface flow visualization technique was used to determine the position of separation lines on the surface of the model and a scanning laser light sheet was used to examine the characteristics of the vortices.

The $\mathrm{TiO}_{2}$ technique involved painting the model with a mixture of $\mathrm{TiO}_{2}$ and kerosene with a small amount of oleic acid added as an anticoagulant. After the mixture was painted on the surface of the model, the airspeed in the test section was brought up to the test condition. The kerosene evaporated and left the $\mathrm{TiO}_{2}$ deposited on the surface. This technique provided the fine-grain detail that was necessary to identify features such as boundary layer separation and attachment lines on the surface of the model. Figures 7 and 8 show the surface pattern on the 
model at $\alpha=15^{\circ}$ and $25^{\circ}$ respectively. At $\alpha=15^{\circ}$ the separation lines on the main wings are symmetrical. There is considerable spanwise flow with reverse flow on the outer wing panel. The secondary separation lines at $\alpha=15^{\circ}$, are clearly defined near the edge of the LEX until the wing/LEX intersection, at which point they appear to be swept outboard. At $\alpha=25^{\circ}$ the main wing separation is no longer symmetrical. The right hand or starboard wing has a much stronger spanwise flow. The secondary separation lines on the LEX have moved inboard away from the edge of the LEX and tend to lose their identity just forward of the wing/LEX intersection.

A scanning laser light sheet was used to determine the location of the vortices over the model and the presence of vortex breakdown. The system was designed by Rhodes (10), et. al. at NASA Langley and can be used in a variety of modes which include either single or simultaneous multiple light sheets. In addition, the system can rotate the sheets through $360^{\circ}$ or provide a single scanning sheet with adjustable scan rates. Smoke was introduced into the flow upstream of the honeycomb, and is illuminated with a thin sheet of laser light to provide a crosssectional view of the flowfield. The smoke was produced by vaporizing propylene glycol at a temperature of $380^{\circ} \mathrm{F}$. The light sheet was generated using a 5 watt Argon-ion laser as the light source with a twinmirrored galvanometer laserlight-sheet generator. Figures 9 and 10 are photographs of the laser light sheet flow visualization over the model at $\alpha=15^{\circ}$ and $25^{\circ}$ respectively. The laser light sheets in the photographs were positioned to the approximate locations of the two survey planes at stations 440 and 524 . The dark hole in the center of the vortices at $\alpha=15^{\circ}$ (figure 9) are characteristic of vortices that are unburst. The center is dark because smoke particles have not been entrained into the confined core region. The trajectory for the LEX vortices at $\alpha=15^{\circ}$ lies close to the model surface and outboard of the twin tails. At $\alpha=25^{\circ}$ (figure 10) the core of the vortices at station 524, just ahead of the twin tails, has become filled with smoke indicating that vortex breakdown has occurred.

The actual breakdown location is in the vicinity of the forward crosssection at station 440. Video pictures of the laser light sheet flow visualization showed the cores of the vortices at station 440 alternating from dark to light indicating that the breakdown point is moving through this plane.

\section{VELOCITY SURVEYS}

The major objective of this investigation was to obtain velocity surveys in the vortex dominated flowfield over an advanced fighter 
configuration at high angles of attack. A typical survey plane consisted of approximately 221 measurement locations and took about 6 hours to acquire. The measurement grid contained typically 17 evenly spaced points spanwise and 13 points vertically. The measurement grid was "body fitted". The surface of the model at specified span stations was determined from a piecewise cubic spline interpolation of the cross sectional data supplied by Northrop. The set of interpolated points formed a "base-survey line" and the grid was constructed by generating new survey lines that were a constant vertical increment from each previous survey line. The lowest survey line was the exception in that it was half the normal vertical increment. This was done to enable one survey to be performed near the surface. In many cases, LDV velocity measurements were not possible at every measurement point in the survey plane, due to scattered light or flare from the laser beams on the model surface. This typically occurred at the lower survey lines; however, there were cases when a occasional point was missed higher in the flowfield due to the laser beams striking screws, mounting holes or production breaks on the model.

The flowfield data in this paper will be shown superimposed over the cross section of the YF-17 at station 524. The vertical tail will also be shown, even though it is aft of this station, to help orient the reader as to the position of the vortices relative to the vertical tail.

The mean velocities that were measured at $\alpha=15^{\circ}$ are shown in figures 11 and 12 . The cross flow velocity vectors are shown in figure 11 and velocity contours for all three components are shown in figure 12 . The cross flow velocity vectors clearly show the LEX vortex is positioned outboard of the vertical tail and close to the wing upper surface. The $v$ (spanwise) component of velocity has accelerated to approximately $90 \%$ of freestream directly under the LEX vortex. The $u$ (axial) component of velocity in the core of the LEX vortex has decelerated to approximately $80 \%$ of freestream.

The flow separates at the leading edge of the wing at this angle of attack. There is a large separated flow region over the outer wing panel at this station. There are reverse flows of up to $10 \%$ of freestream (figure 12a) in the axial velocity component over the outer third of the wing span. The LEX vortex had the favorable effect of preventing wing separation over the inboard portion of the wing. The flow angularity $\theta$ shown in figure 13 , reaches a maximum of approximately $40^{\circ}$ and $80^{\circ}$ in the LEX vortex and wing separation regions respectively. The higher $\theta$ values in the wing separation region are primarily due to the low axial velocities in this region. 
The flowfield takes on a dramatically different character at $25^{\circ}$ angle of attack. The mean velocity vectors and contours are shown in figures 14 and 15 respectively. The cross flow velocity vectors (figure 14) show that the wing separation region and the LEX vortex have grown in size and the LEX vortex is positioned directly in line with the vertical tail. The LEX vortex has burst further forward near station 440 at this angle of attack. The core of the LEX vortex contains a large area of reversed flow as shown in figure $15 \mathrm{a}$. The reversed flow reaches a maximum of approximately $10 \%$ of freestream. Although the LEX vortex has burst, there is still a structured swirling pattern around the vortex. This is consistent with the measurements of a burst vortex over $75^{\circ}$ delta wing reported in (6); however, the flowfield is more complicated due to the presence of the wing separation which has merged with the flow from the LEX vortex. The region of reverse flow in the wing separation region has grown in both size and strength with the magnitude of the reverse flow increasing to approximately $20 \%$ of freestream. Figure 16 shows that the total flow angles near the core of the LEX vortex have increased to approximately $70^{\circ}$. The size of the region of high total flow angles due to wing separation has also increased.

The RMS of the velocity fluctuations provides a measure of the intensity of the fluctuating input forcing the vertical tail to vibrate. The equation that was used to calculate the RMS of the $u$ component is shown below:

$$
\operatorname{RMS}=\sqrt{\frac{u_{i} \bar{u}^{2}}{N}}
$$

Where $N$ is the number of particle velocity measurements at a given location. The RMS is routinely calculated by the LDV system and is nondimensionalized by the freestream velocity to provide a measure of the relative turbulence intensity. The remaining figures will present the RMS in terms of percent of freestream. The RMS for all three velocity components is presented in figures 17 and 18 for $\alpha=15^{\circ}$ and $25^{\circ}$ respectively. At $\alpha=15^{\circ}$, most of the larger values for the velocity fluctuations are confined to the wing separation region and the LEX vortex. This fact is most evident for the $v$ component as shown in figure $17 \mathrm{~b}$. The maximum values for the RMS velocity fluctuations at $\alpha=15^{\circ}$ reach levels of approximately $39 \%, 31 \%$, and $26 \%$ for the $u, v$, and $w$ components respectively. At $\alpha=25^{\circ}$, the turbulent region has grown considerably in size. The maximum value for the RMS velocity fluctuations reach levels of approximately $40 \%, 35 \%$ and $30 \%$ for the $u$, $v$, and $w$ component respectively. Although the maximum values for the RMS velocity fluctuations have not grown considerably, their region of influence has. The large RMS values in the upper portion of the wing separation region are due to movement of the shear layer through the 
measurement location. The RMS levels for the $v$ component have increased from $2 \%$ of freestream at $\alpha=15^{\circ}$, to approximately $30 \%$ at the midspan position of the vertical tail.

\section{SUMMARY}

A study was conducted of the vortex dominated flowfield over a YF-17 model at two angles of attack. A three component Laser Doppler Velocimeter was used to measure the time-average and root-meansquare of the velocity components at a station just ahead of the twin tails. At $\alpha=15^{\circ}$ the vortices generated by the LEXs are unburst and pass outboard of the base of the vertical tail. At $\alpha=25^{\circ}$ the LEX vortices burst in the vicinity of the wing/LEX intersection and are positioned directly in line with the vertical tails. The core of the LEX vortex includes a region of reverse flow that ranges up to $10 \%$ of freestream. The maximum values for the RMS velocity fluctuations reach levels of $40 \%, 35 \%$, and $30 \%$ for the $u, v$, and $w$ components respectively.

\section{ACKNOWLEDGEMENT}

The authors wish to express their appreciation to the Aircraft Divison of the Northrop Corporation, for the use of the YF-17 model used during this investigation and for their kind assistance during this test program. The authors wish to acknowledge in particular, the assistance of Mr. A. P. Humphreys.

\section{NOMENCLATURE}

Values are given in U.S. Customary Units, often with equivalent values in the International System of Units (SI) given in parenthesis for the convenience of readers. Measurements and calculations were made in U.S. Customary Units.

$\begin{array}{ll}L & \text { characteristic length } \\ R_{n} & \text { Reynolds number, } V_{\infty} L / \\ S_{t} & \text { Stokes number } \\ q & \text { dynamic pressure, } \rho V_{\infty}^{2} / 2 \\ V_{\infty} & \text { freestream velocity, } \mathrm{ft} / \mathrm{sec}\end{array}$




$$
\begin{array}{ll}
u, v, w \quad \begin{array}{l}
\text { streamwise, lateral and vertical velocity components in the } \\
\text { body-axis system, } \mathrm{ft} / \mathrm{sec}
\end{array} \\
\begin{array}{ll}
x, y, z \quad & \begin{array}{l}
\text { streamwise, lateral and vertical coordinates in body axis } \\
\text { system, inches }
\end{array} \\
\alpha \quad & \text { angle of attack, deg } \\
& \text { kinematic viscosity, } \mathrm{ft}^{2} / \mathrm{sec} \\
& \text { density, slug } / \mathrm{ft}^{3}
\end{array} \\
\sigma \quad \text { standard deviation } \\
\theta \quad \text { total flow angle, } \sin ^{1} \frac{\sqrt{v^{2} w^{2}}}{\sqrt{u^{2} v^{2} w^{2}}}
\end{array}
$$

Subscripts:

at free stream conditions

Superscripts:
$\sim \quad$ root-mean-square value of the fluctuating component of velocity
- $\quad$ time-averaged or mean

Abbreviations:

BART Basic Aerodynamics Research Tunnel

LDV Laser Doppler Velocimeter

LVABI laser velocimeter autocovariance buffer interface

MAC mean aerodynamic chord

RMS root-mean-square

\section{REFERENCES}

1. Murri, Daniel G. and Rao, Dhanvada M: Exploratory Studies of Actuated Forebody Strakes for Yaw Control at High Angles of 
Attack AIAA-87-2557, AIAA Atmospheric Flight Mechanics Conference, Monterey, CA., August 1987.

2. Triplett, William E.: Pressure Measurements on Twin Vertical Tails in Buffeting Flow, Journal of Aircraft, Vol. 20, No. 11, November 1983.

3. Wentz, William H., Jr.: Vortex-Fin Interaction on a Fighter Aircraft, AIAA-87-2474, AIAA 5th Applied Aerodynamics Conference, Monterey, CA., August 1987.

4. Lan, Edward C. and Lee, I. G.: Investigations of Empennage Buffeting, NASA CR-179426, January 1987.

5. Sellers, William L. III and Kjelgaard, Scott O.: The Basic Aerodynamics Research Tunnel - A Facility Dedicated to Code Validation, AIAA-88-1997, AIAA 15th Aerodynamic Testing Conference, San Diego, CA., May 1988.

6. Meyers, James F. and Hepner, Timothy E.: Measurement of Leading Edge Vortices From a Delta Wing Using a Three Component Laser Velocimeter, AIAA-88-2024, AIAA 15th Aerodynamic Testing Conference, San Diego, CA., May 1988.

7. Nichols, Cecil E., Jr.: Preparation of Polystyrene Microspheres for Laser Velocimetry in Wind Tunnels, NASA TM-89163, June 1987.

8. Dring, R. P. and Suo, M.: Particle Trajectories in Swirling Flows, Journal of Energy, Vol. 2, No. 4, July-August 1978.

9. Cavone, A. A, Sterlina, P. S., Clemmons, J. I., Jr., and Meyers, J. F.: A High-Speed Buffer for LV Data Acquisition, Proceedings of the Second International Symposium on Applications of Laser Anemometry to Fluid Mechanics, Lisbon, Portugal, paper 2.1, 1984.

10. Rhodes, David B., Franke, John M., Jones, Stephen B., Leighty, Bradley D.: A Twin-Mirrored Galvanometer Laser Light Sheet Generator, NASA TM 100587, 1988. 


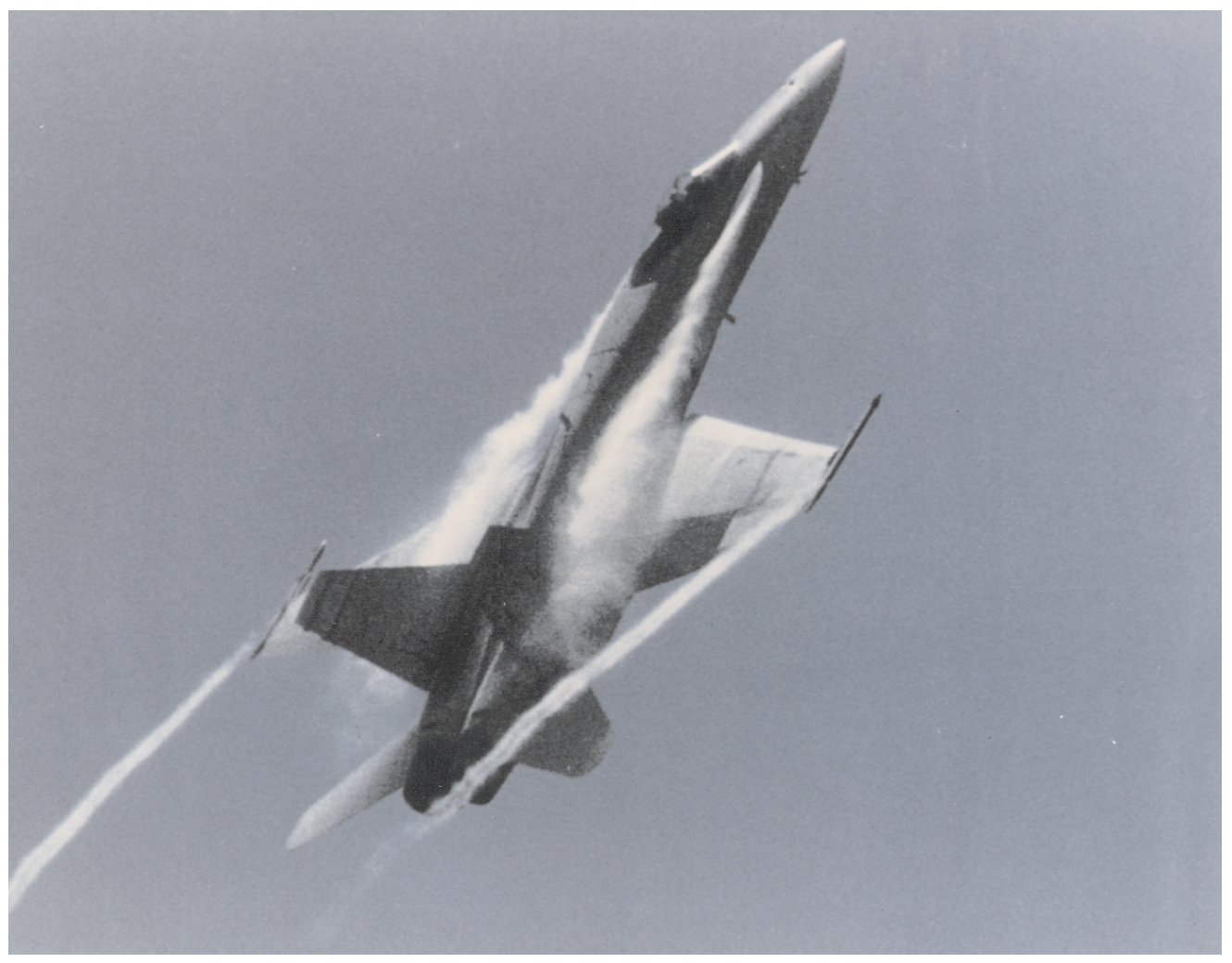

Figure 1. Photograph of an F-18 fighter at high angle of attack.

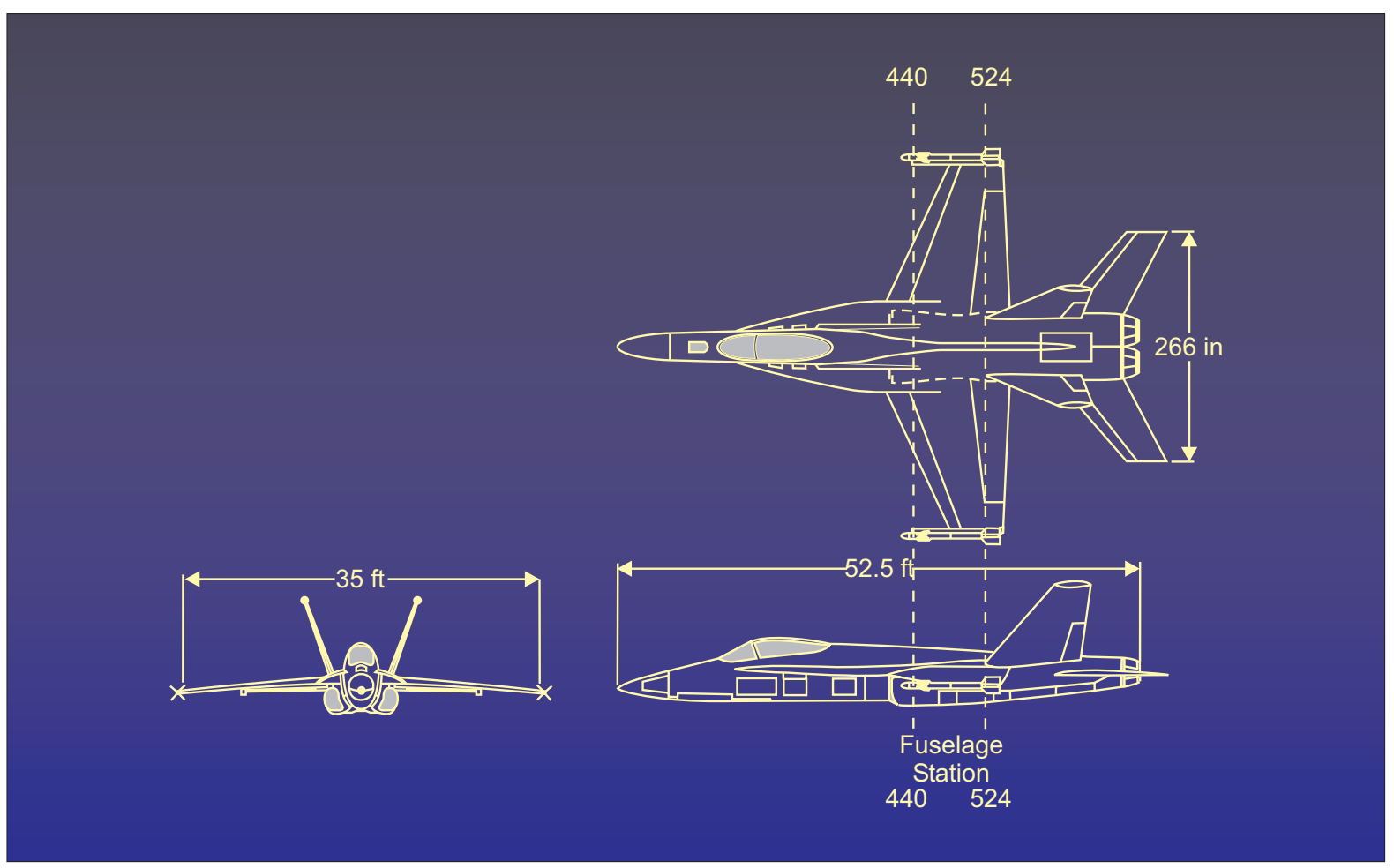

Figure 2. Three-view sketch of the YF-17 configuration. 


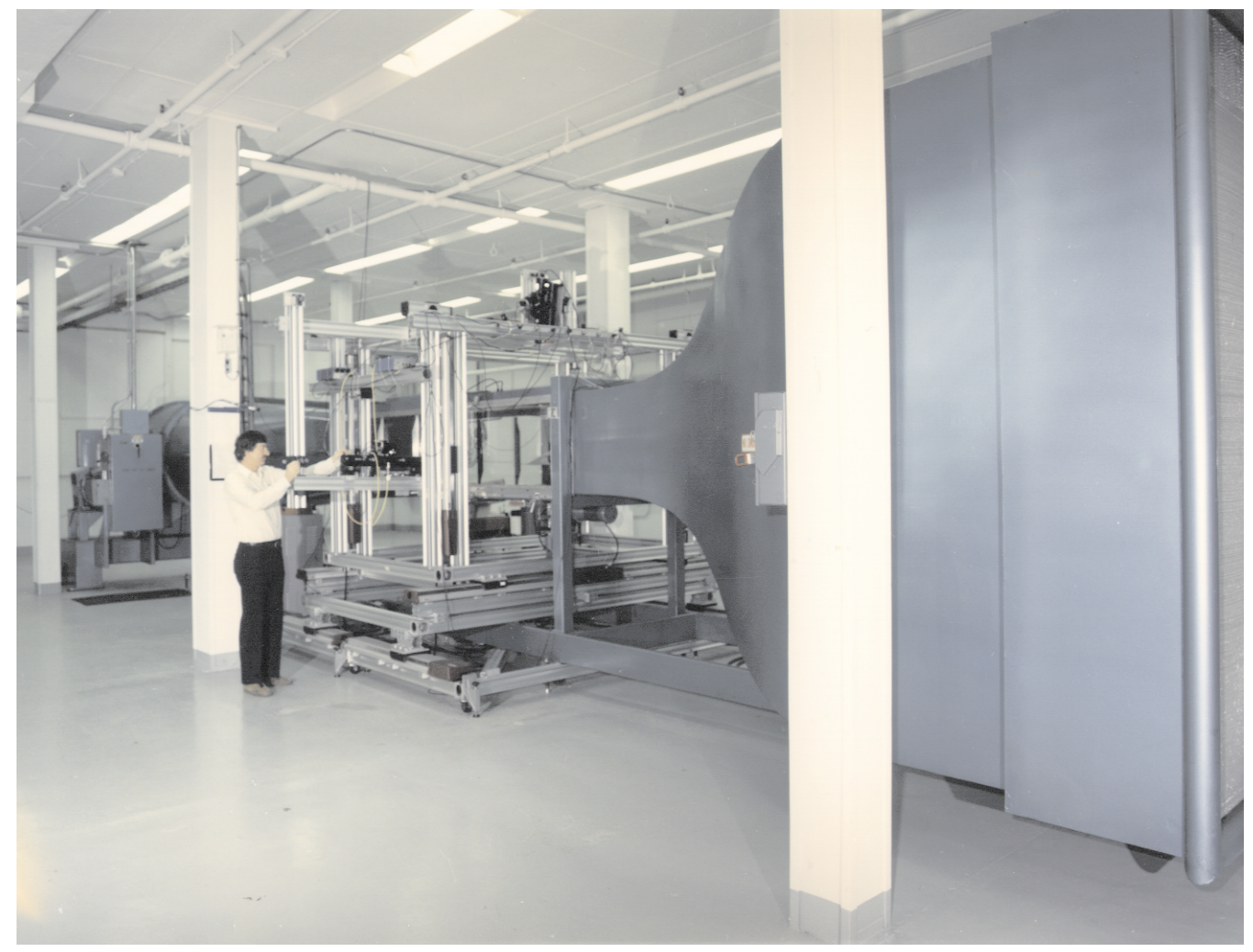

Figure 3. Photograph of the Basic Aerodynamics Research Tunnel.

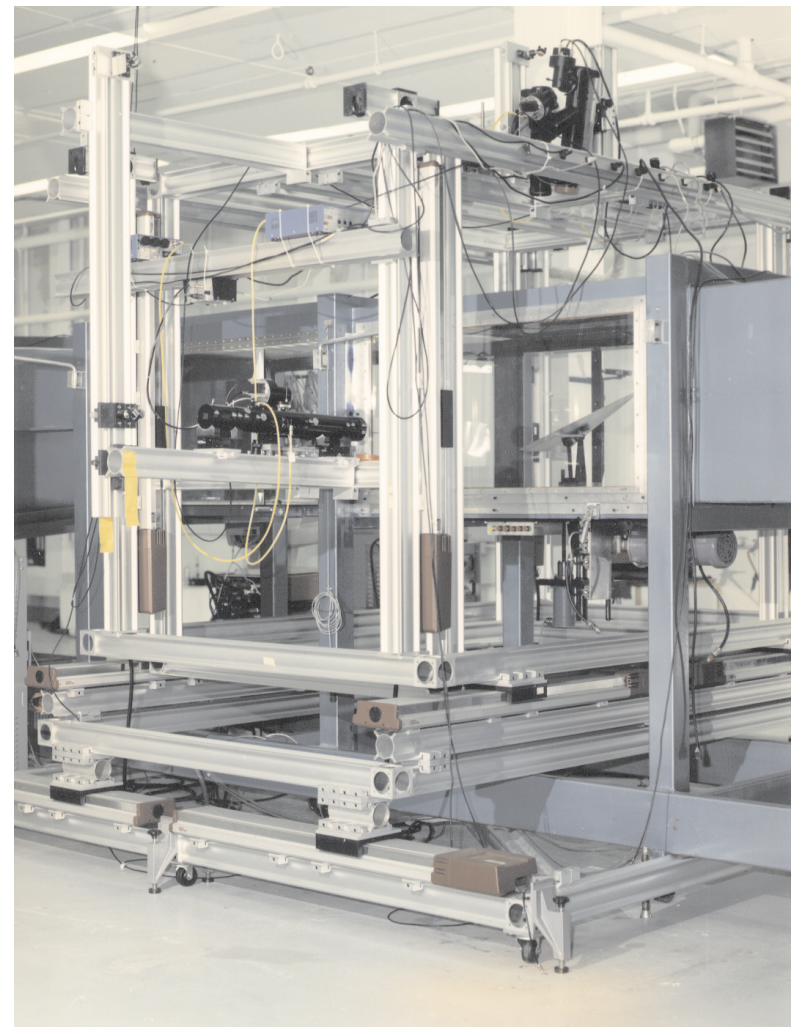

Figure 4. Photograph of the 3-component LDV traversing system. 


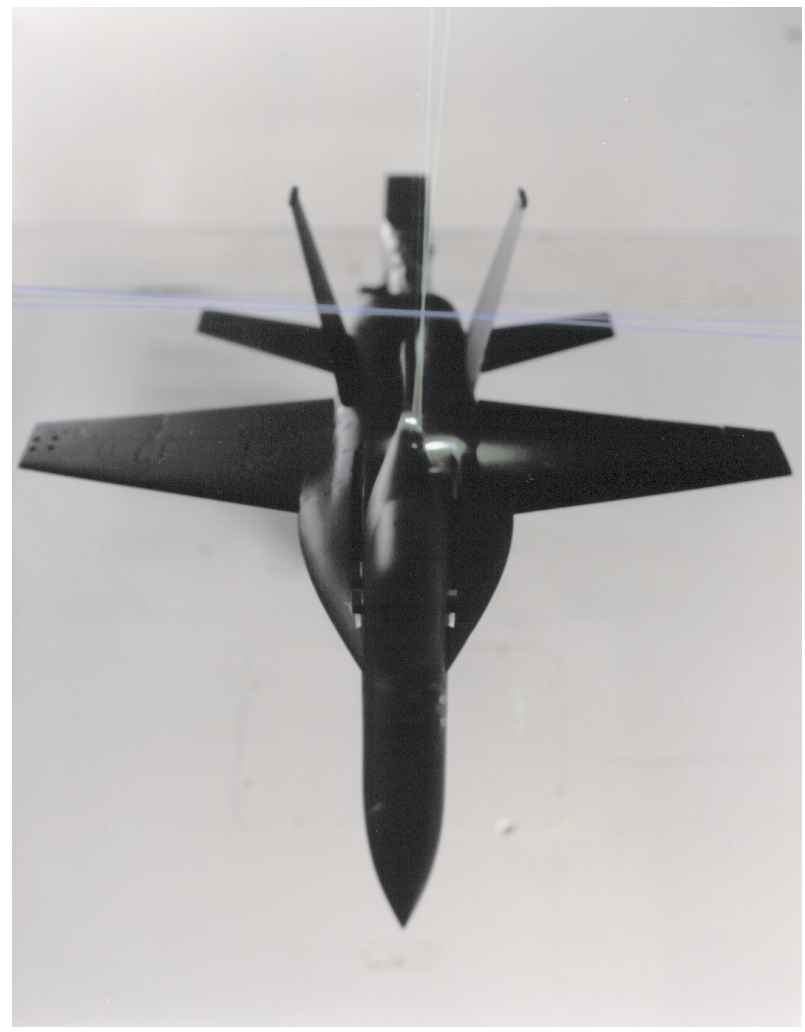

Figure 5. Photograph of the laser beams crossing over the YF-17 model.

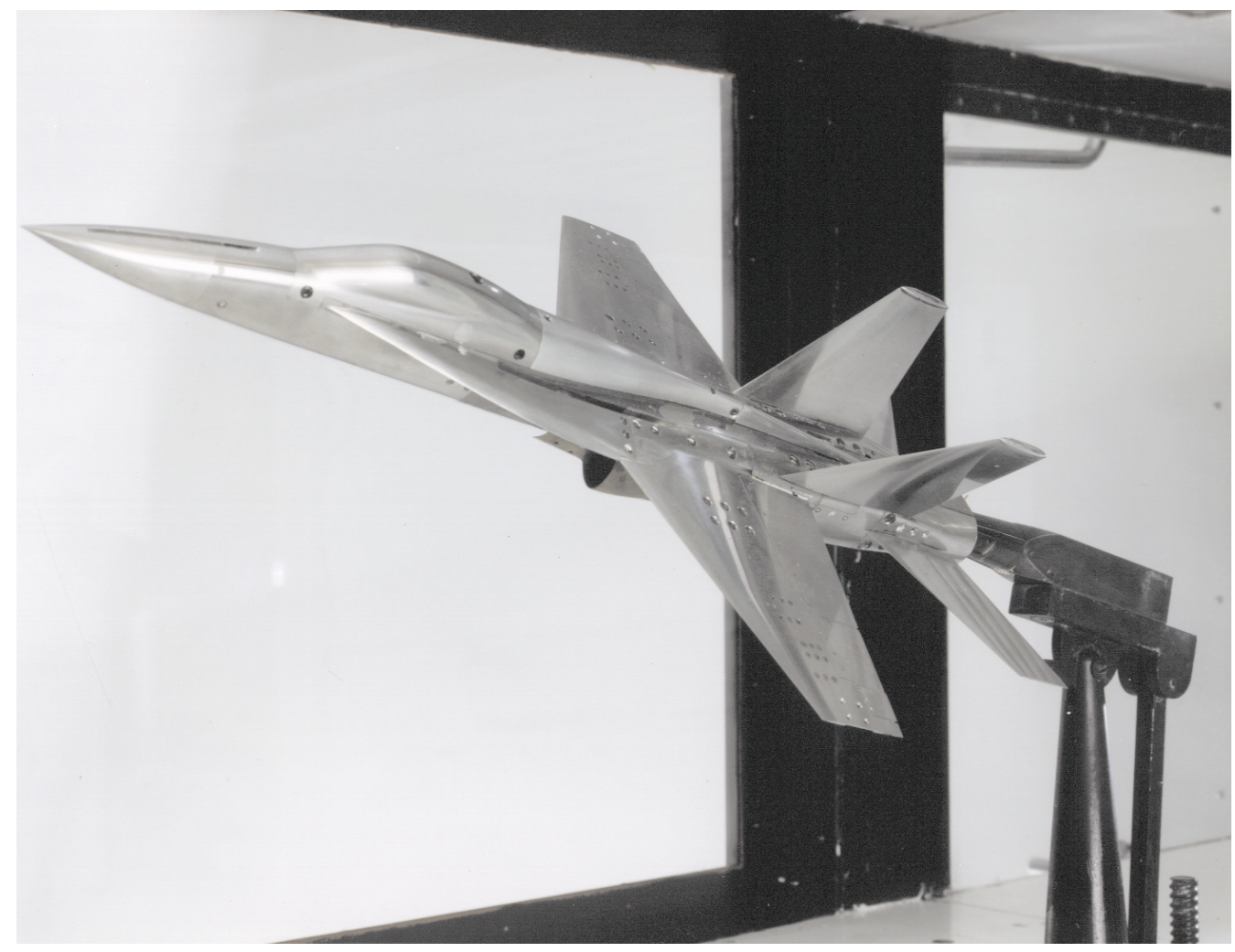

Figure 6. Photograph of the YF-17 model installed in the tunnel. 


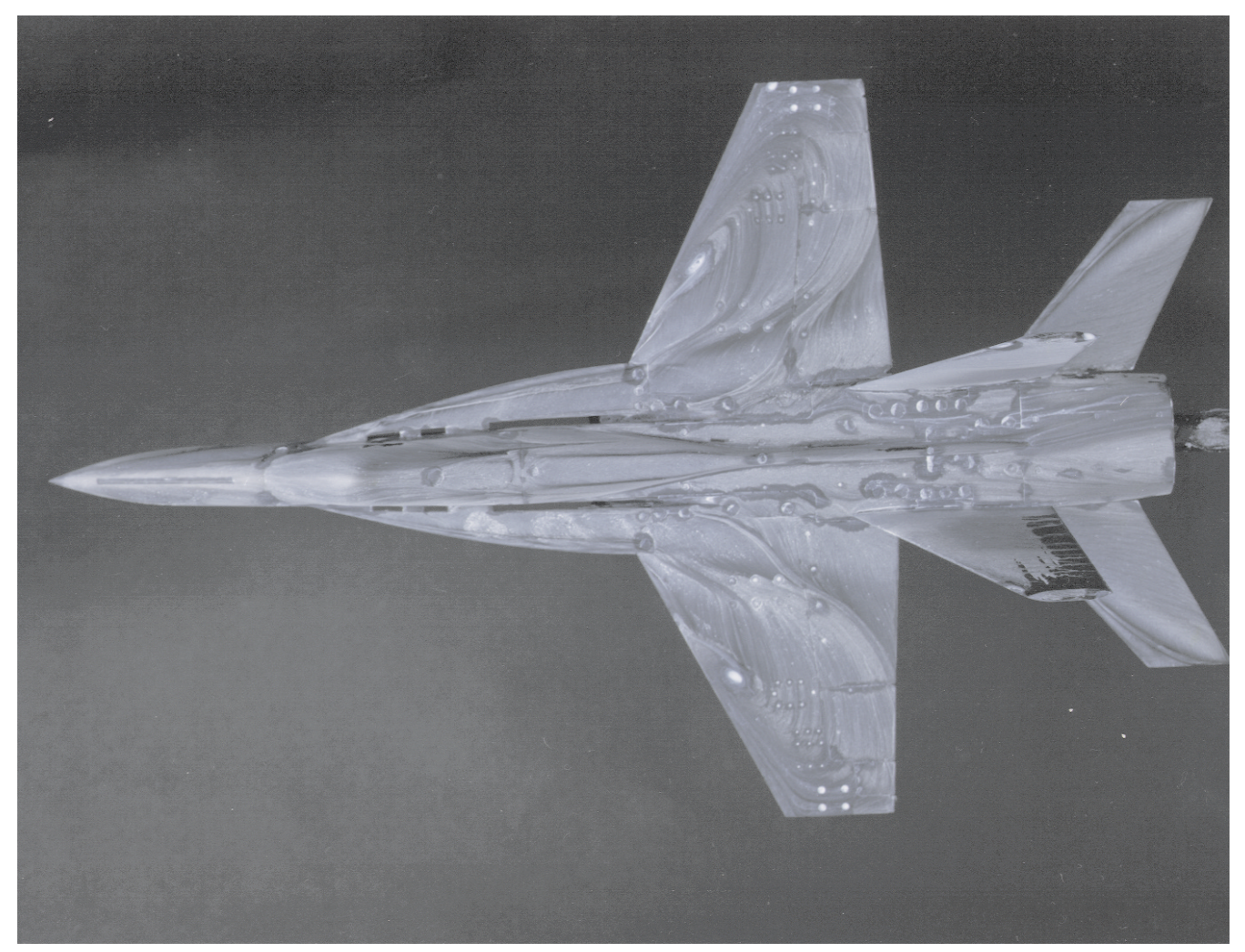

Figure 7. Surface flow visualization; $\alpha=15^{\circ}, R_{n}=326,000$.

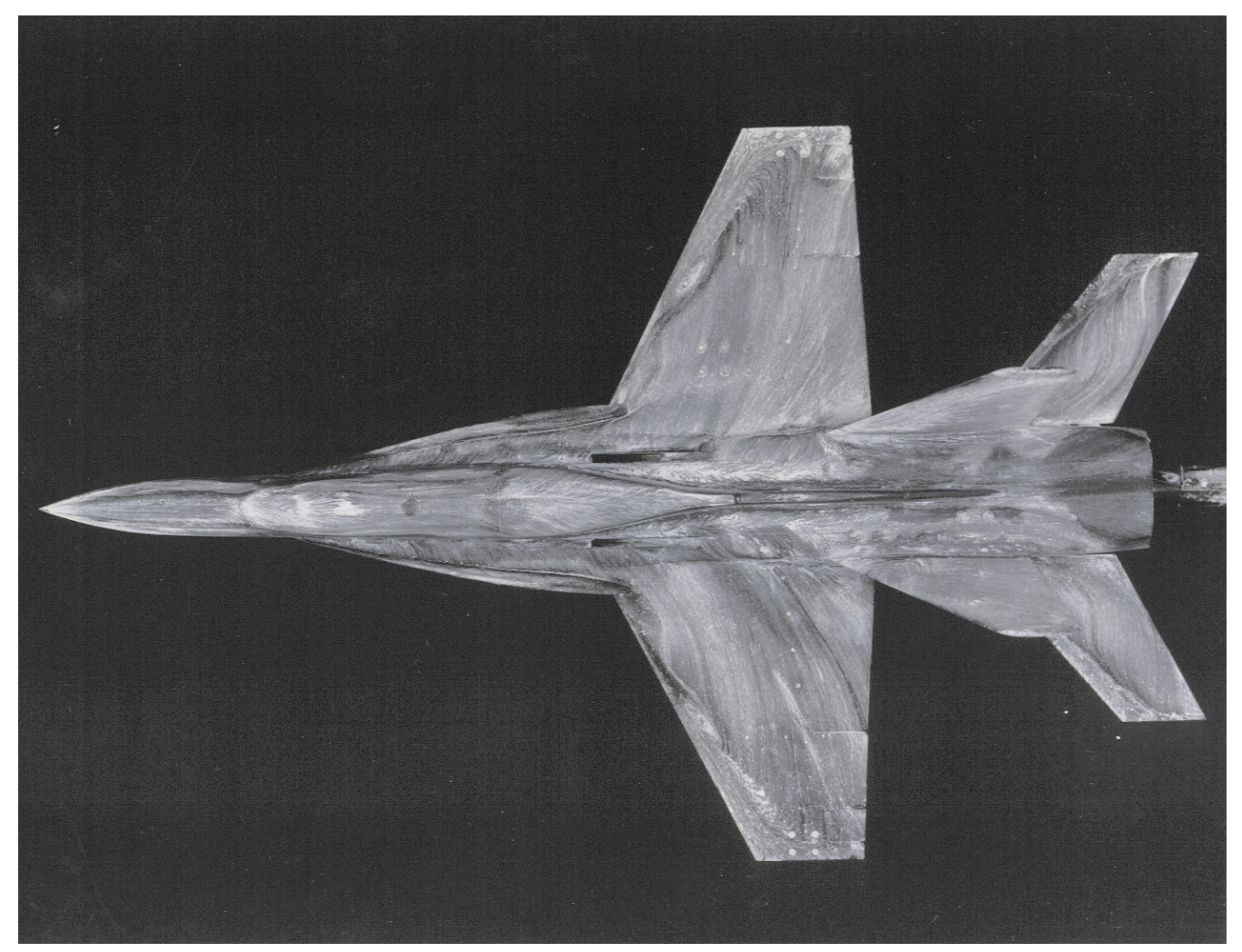

Figure 8. Surface flow visualization; $\alpha=25^{\circ}, R_{n}=326,000$. 


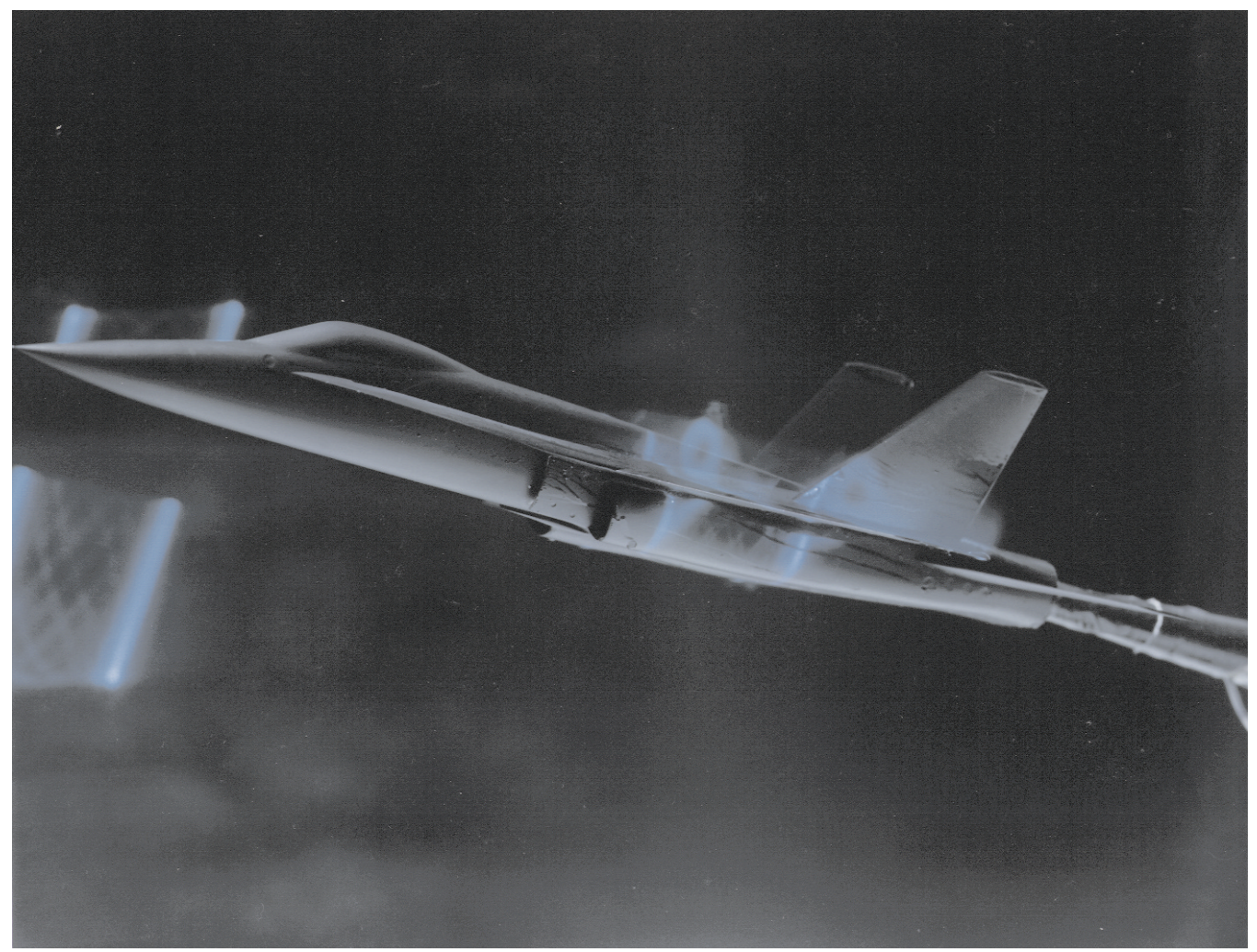

Figure 9. Laser light sheet flow visualization; $\alpha=15^{\circ}$.

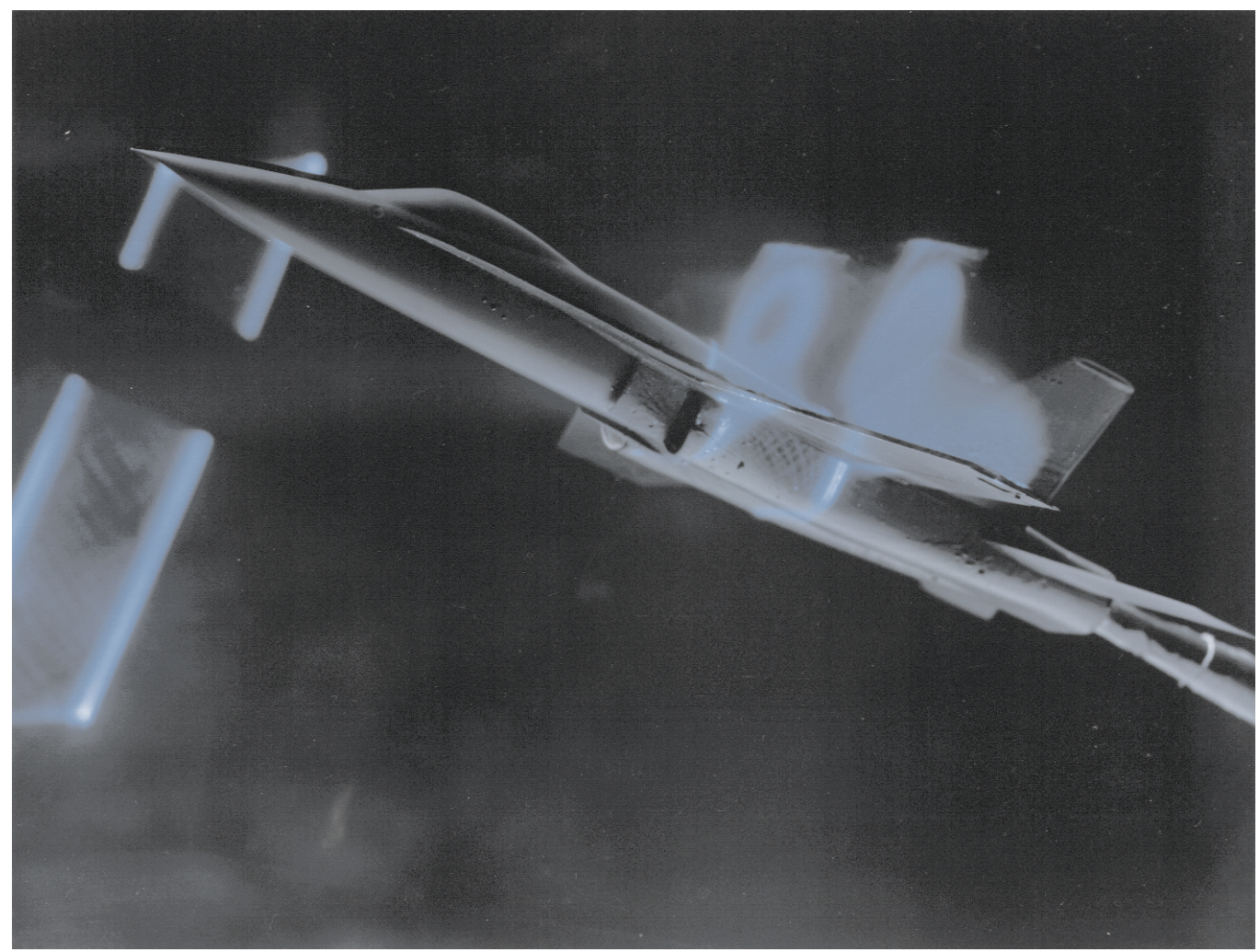

Figure 10. Laser light sheet flow visualization; $\alpha=25^{\circ}$. 


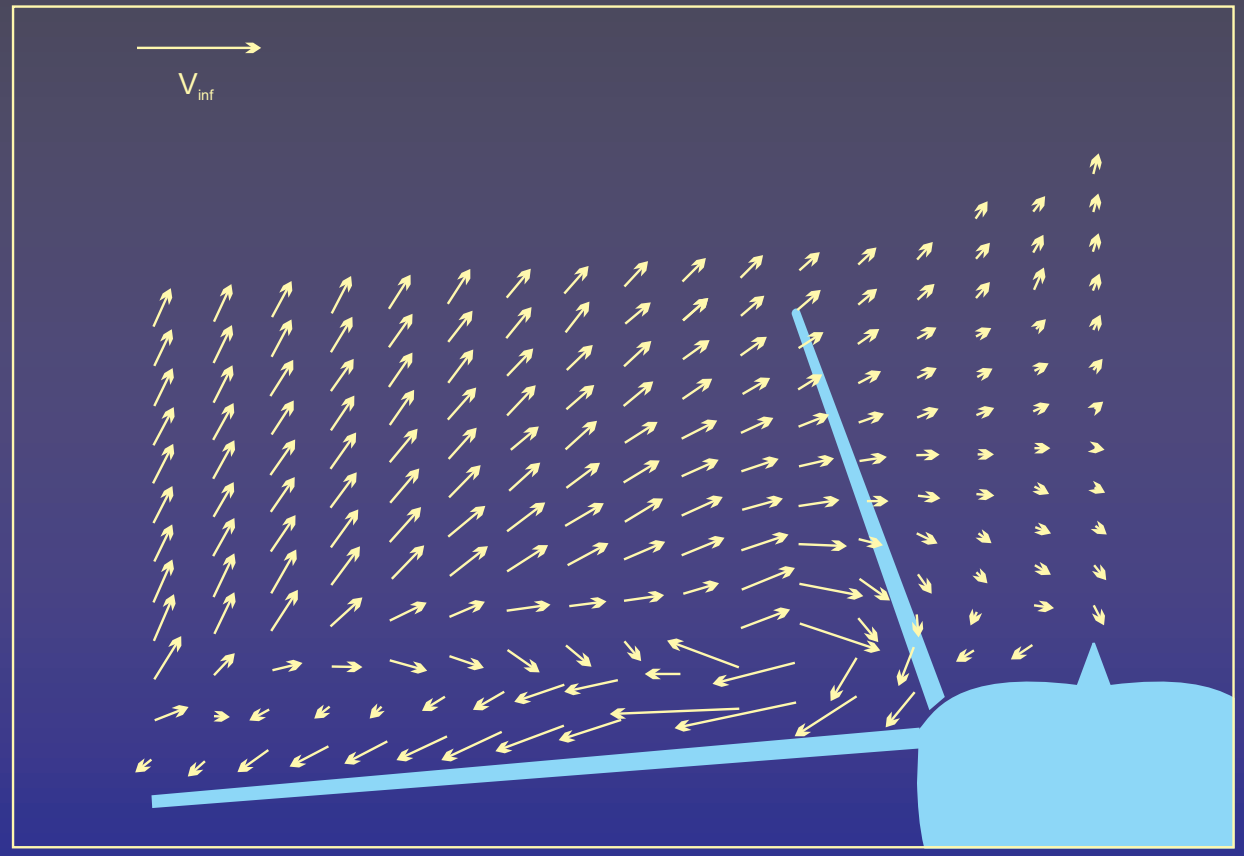

Figure 11. Cross flow velocity vectors, station 524; $\alpha=15^{\circ}, R_{n}=326,000$.

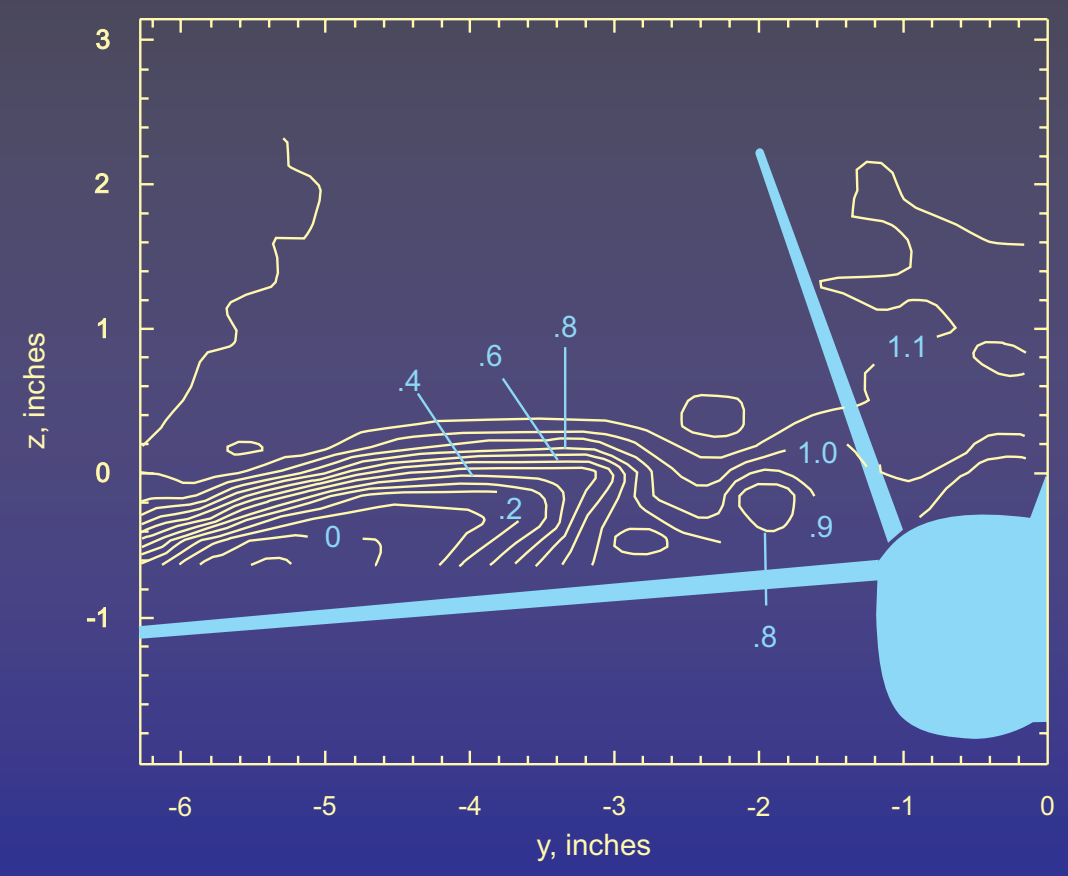

Figure $12 \mathrm{a}$. Contours of mean velocity, $\bar{u} / V_{\infty}$, station 524 ; $\alpha=15^{\circ}, R_{n}=326,000$. 


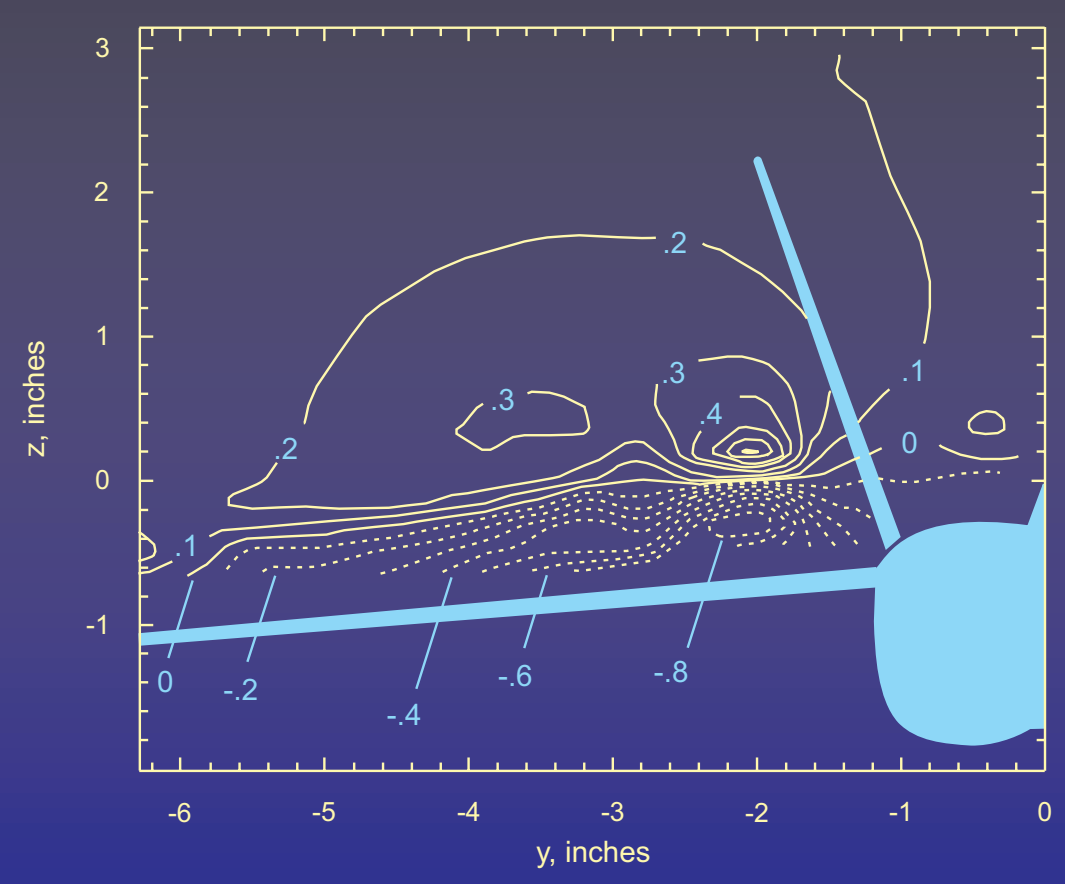

Figure $12 \mathrm{~b}$. Contours of mean velocity, $\bar{v} / V_{\infty}$, station 524 ; $\alpha=15^{\circ}, R_{n}=326,000$.

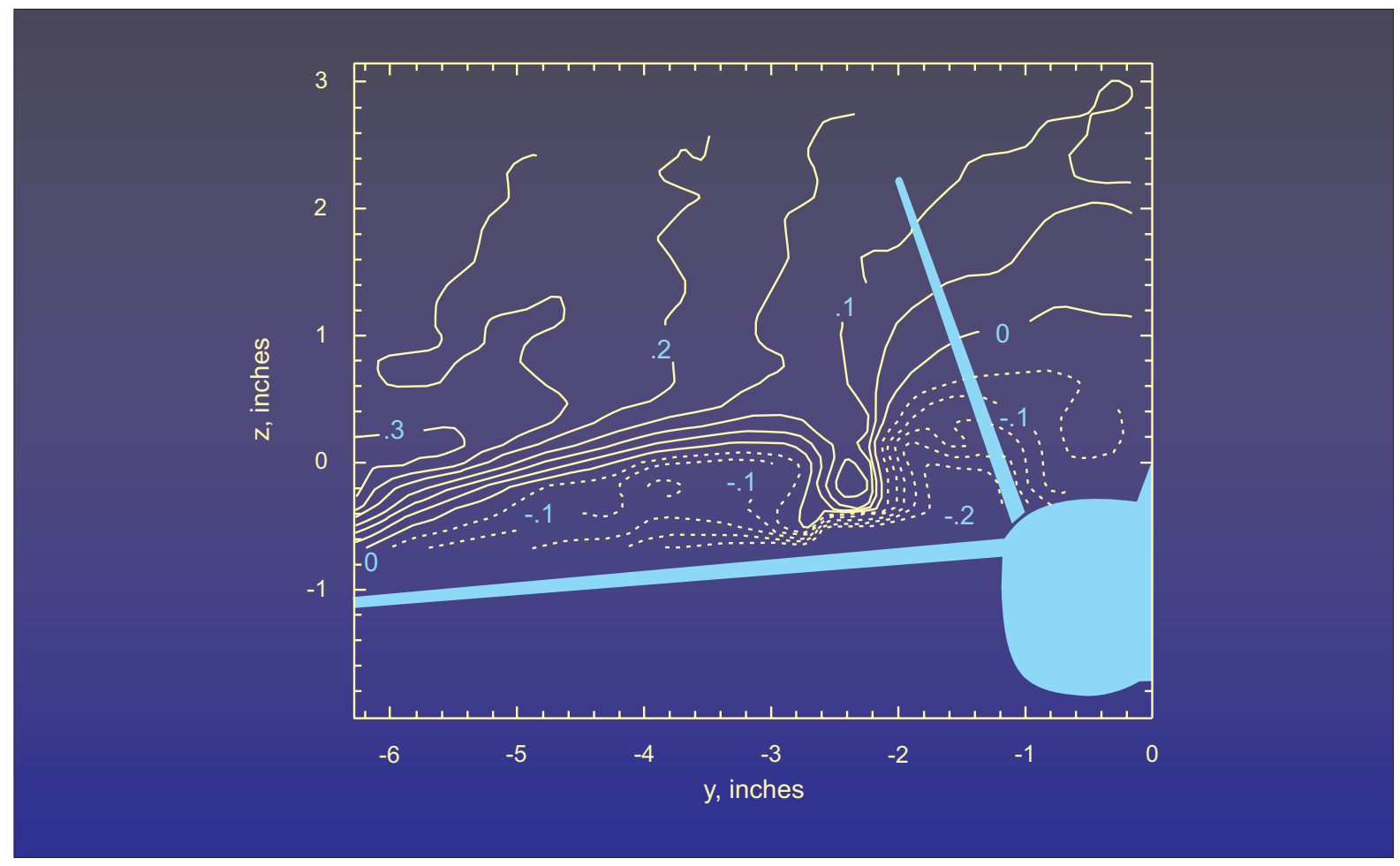

Figure 12c. Contours of mean velocity, $\bar{w} / V_{\infty}$, station 524;

$$
\alpha=15^{\circ}, R_{n}=326,000 \text {. }
$$




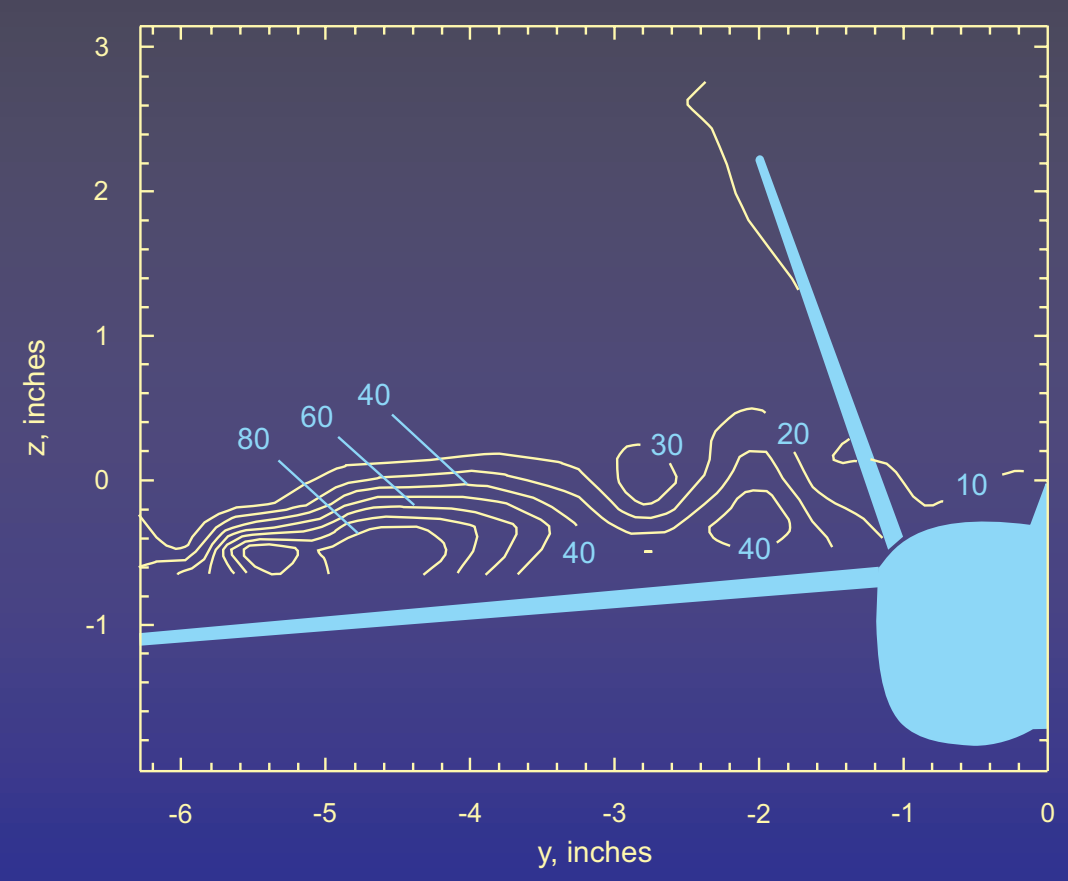

Figure 13. Contours of total flow angle ( $\theta$, in degrees); $\alpha=15^{\circ}$.

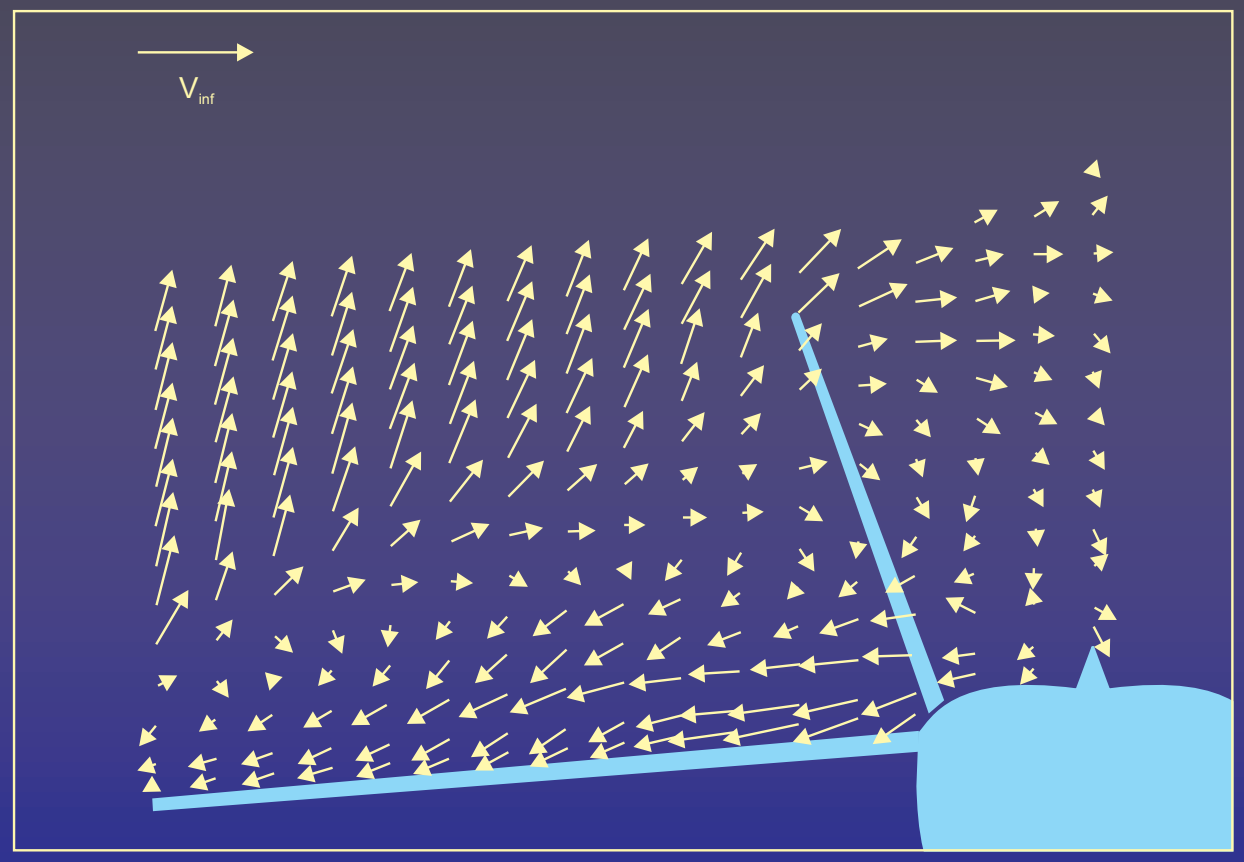

Figure 14. Cross flow velocity vectors, station 524; $\alpha=25^{\circ}, R_{n}=326,000$. 


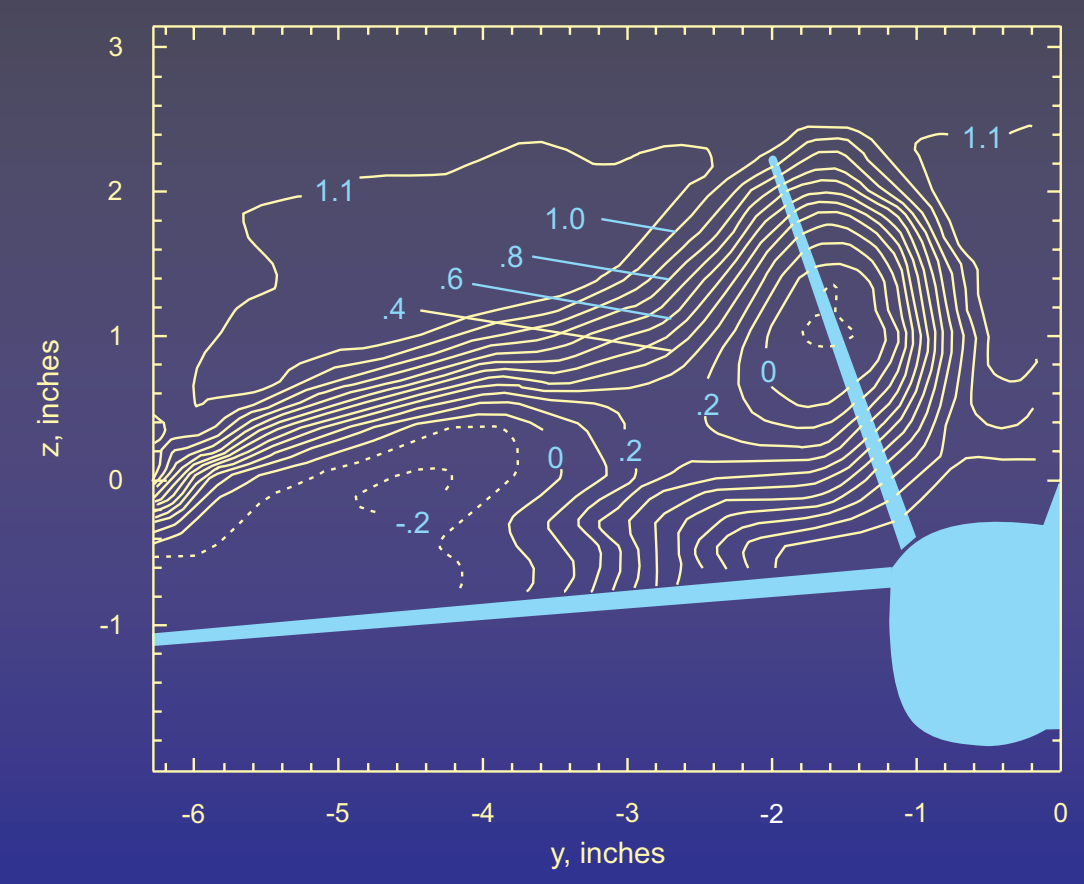

Figure $15 \mathrm{a}$. Contours of mean velocity, $\bar{u} / V_{\infty}$, station 524 ; $\alpha=25^{\circ}, R_{n}=326,000$.

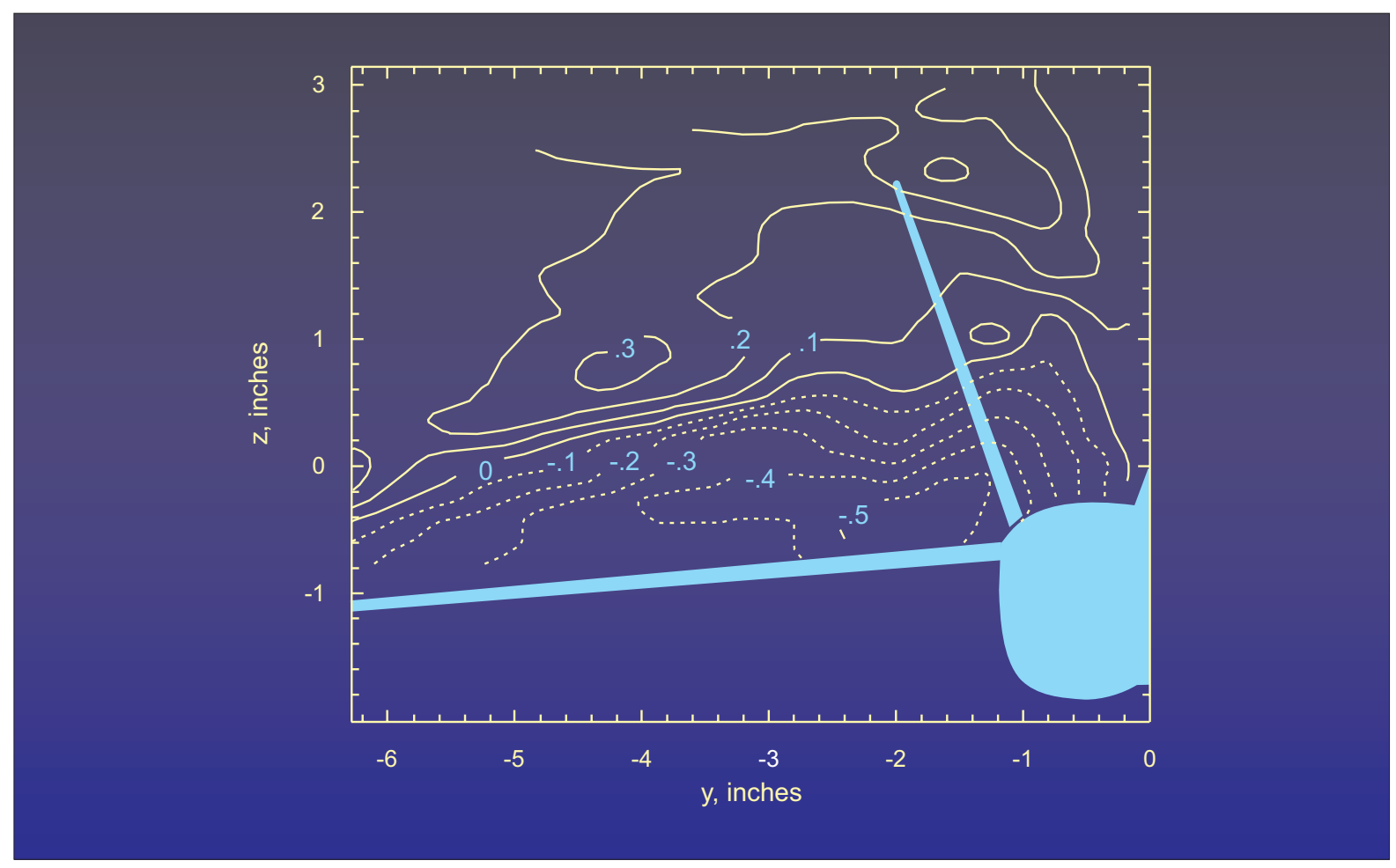

Figure $15 \mathrm{~b}$. Contours of mean velocity, $\bar{v} / V_{\infty}$, station 524 ; $\alpha=25^{\circ}, R_{n}=326,000$. 


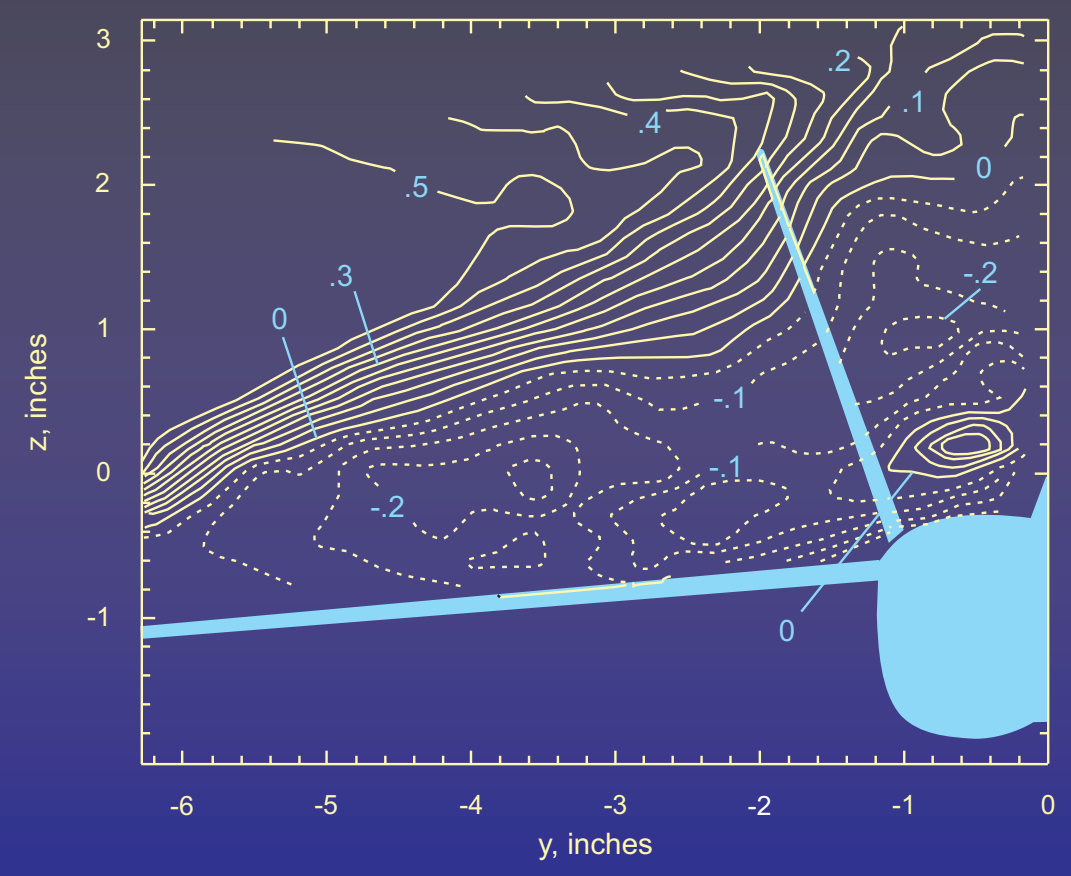

Figure $15 \mathrm{c}$. Contours of mean velocity, $\bar{w} / V_{\infty}$, station 524; $\alpha=25^{\circ}, R_{n}=326,000$.

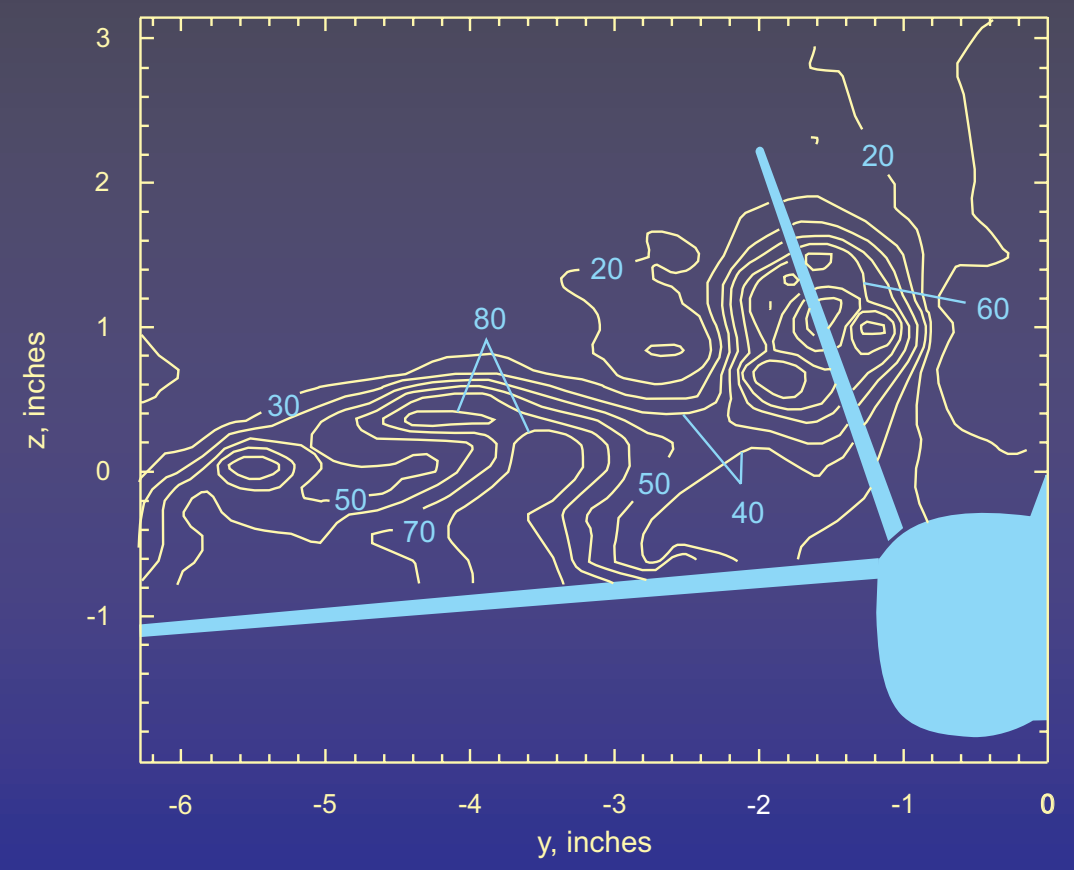

Figure 16. Contours of total flow angle $(\theta$, in degrees $) ; \alpha=25^{\circ}$. 


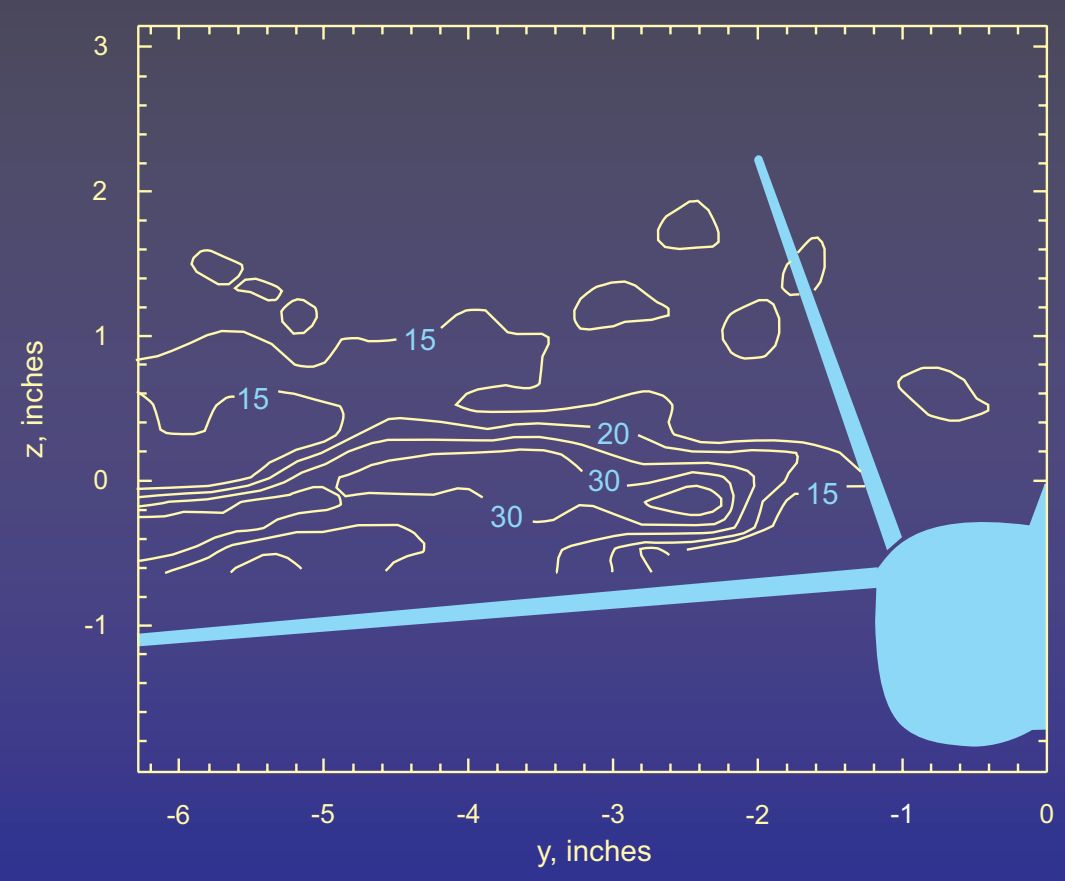

Figure 17a. RMS velocity contours, $\tilde{u} / V_{\infty}$, percent, station 524; $\alpha=15^{\circ}, R_{n}=326,000$.

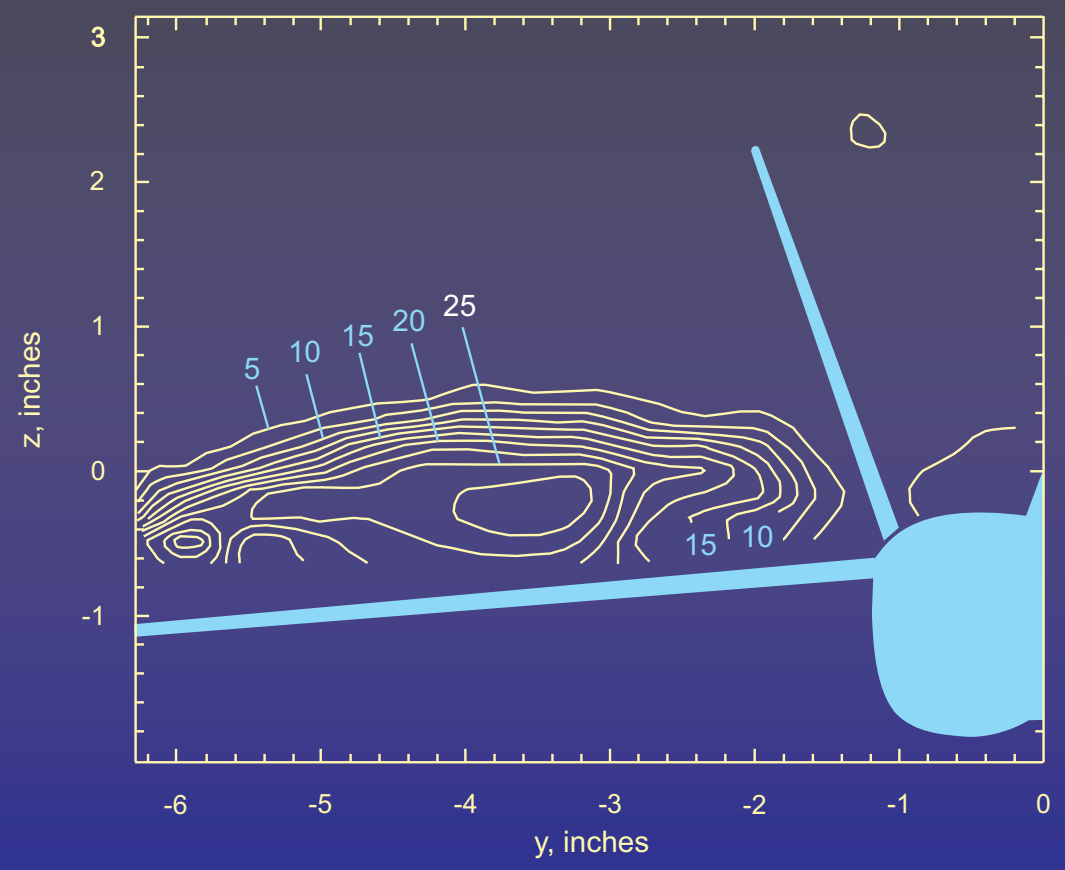

Figure 17b. RMS velocity contours, $\widetilde{v} / V_{\infty}$, percent, station 524; $\alpha=15^{\circ}, R_{n}=326,000$. 


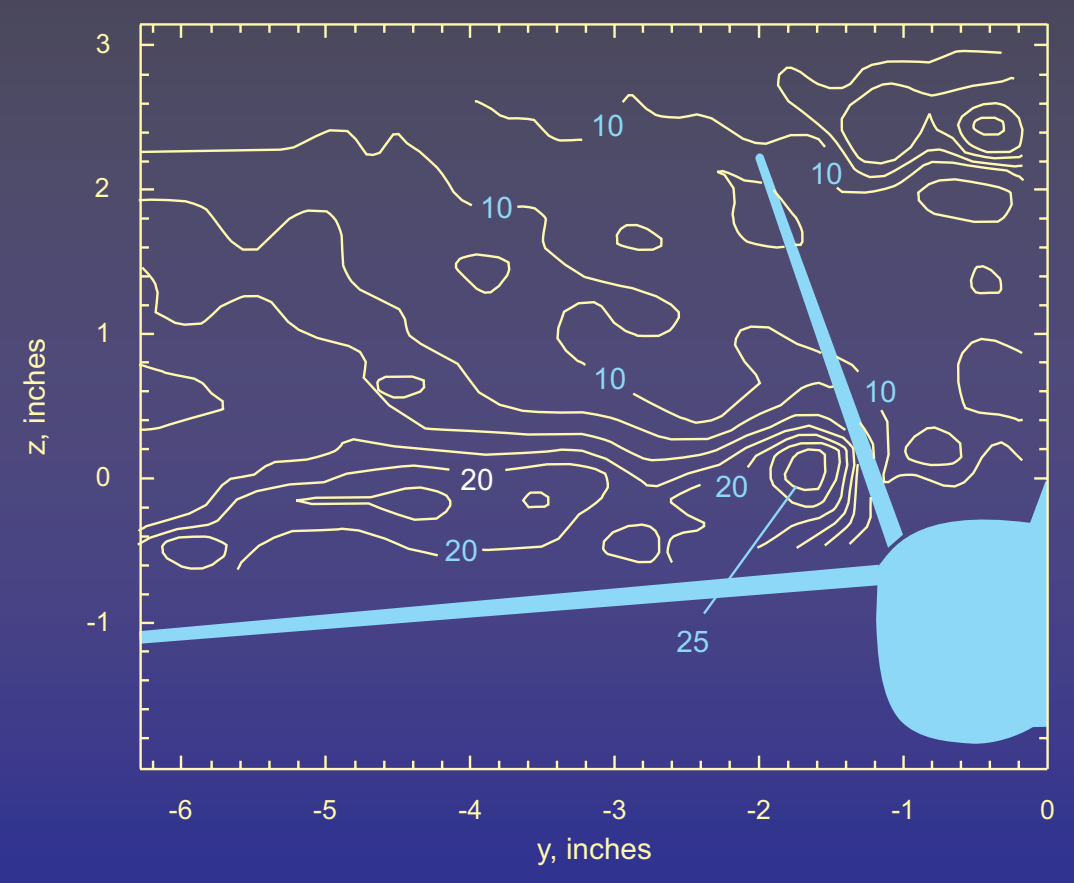

Figure 17c. RMS velocity contours, $\widetilde{w} / V_{\infty}$, percent, station 524; $\alpha=15^{\circ}, R_{n}=326,000$.

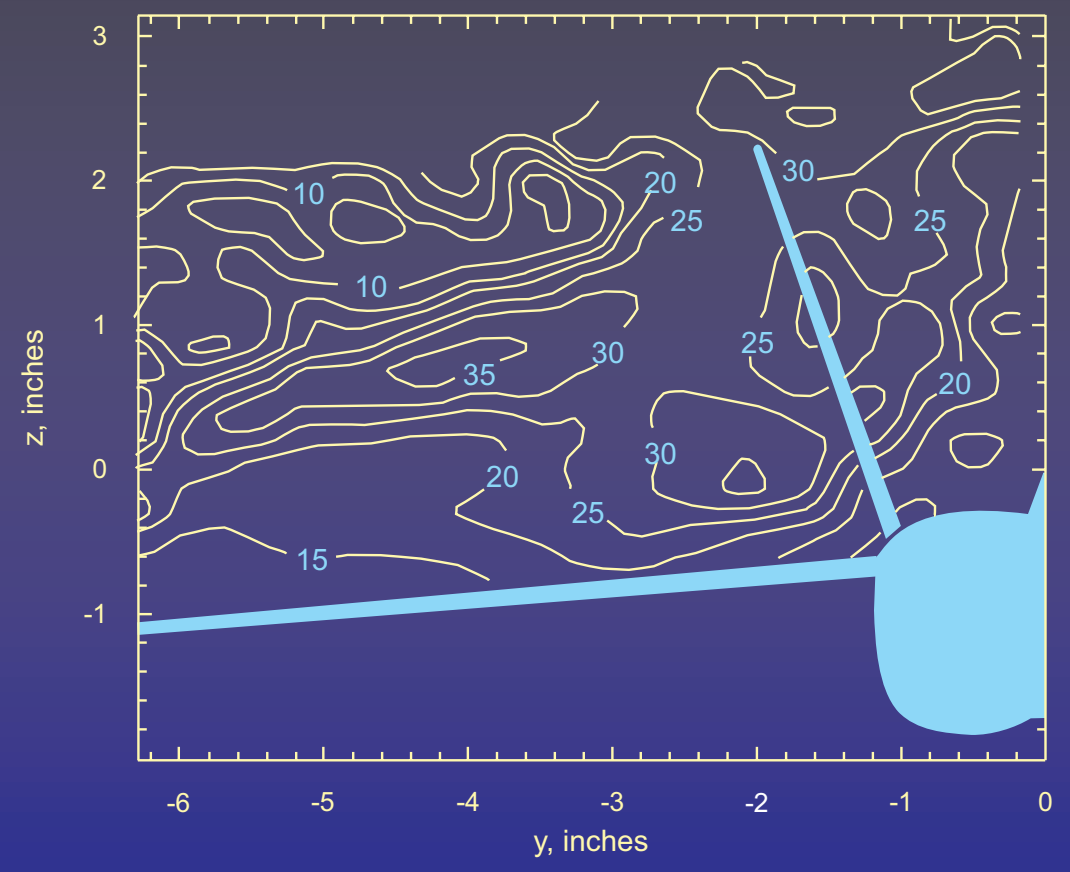

Figure 18a. RMS velocity contours, $\widetilde{u} / V_{\infty}$, percent, station 524;

$$
\alpha=25^{\circ}, R_{n}=326,000 \text {. }
$$




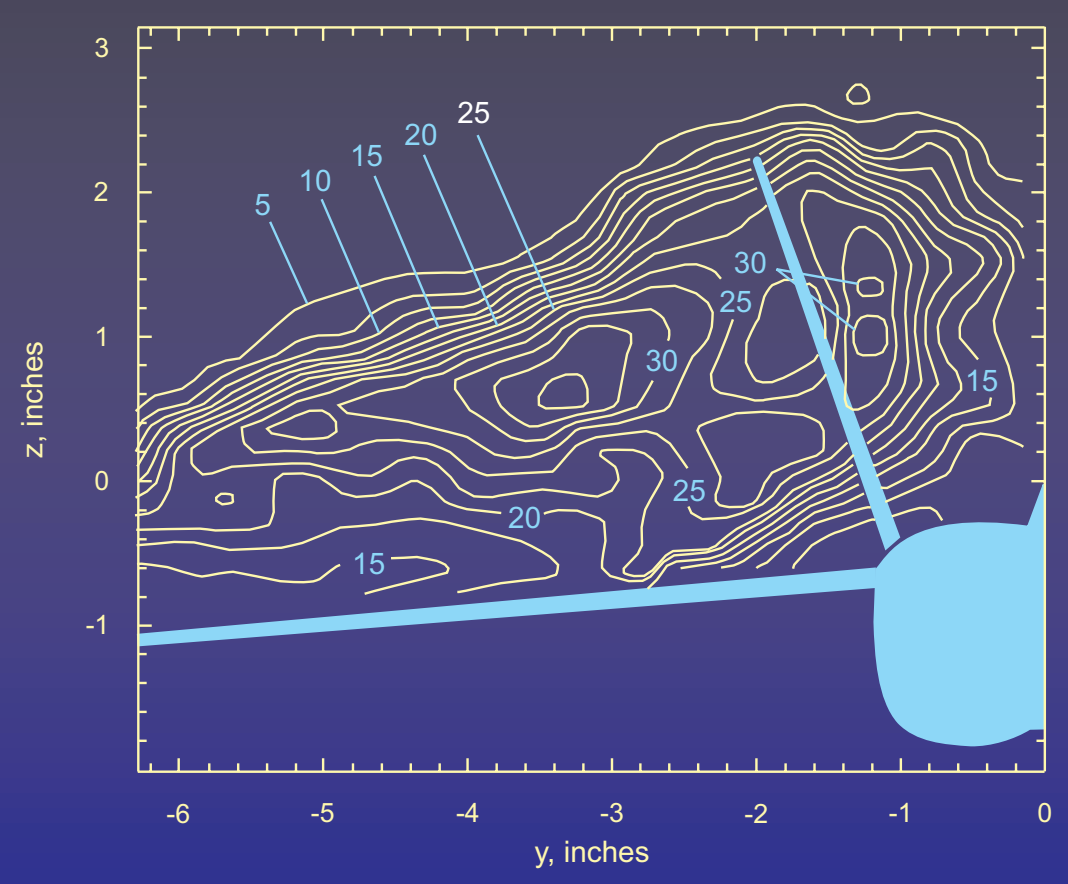

Figure 18b. RMS velocity contours, $\widetilde{v} / V_{\infty}$, percent, station 524; $\alpha=25^{\circ}, R_{n}=326,000$.

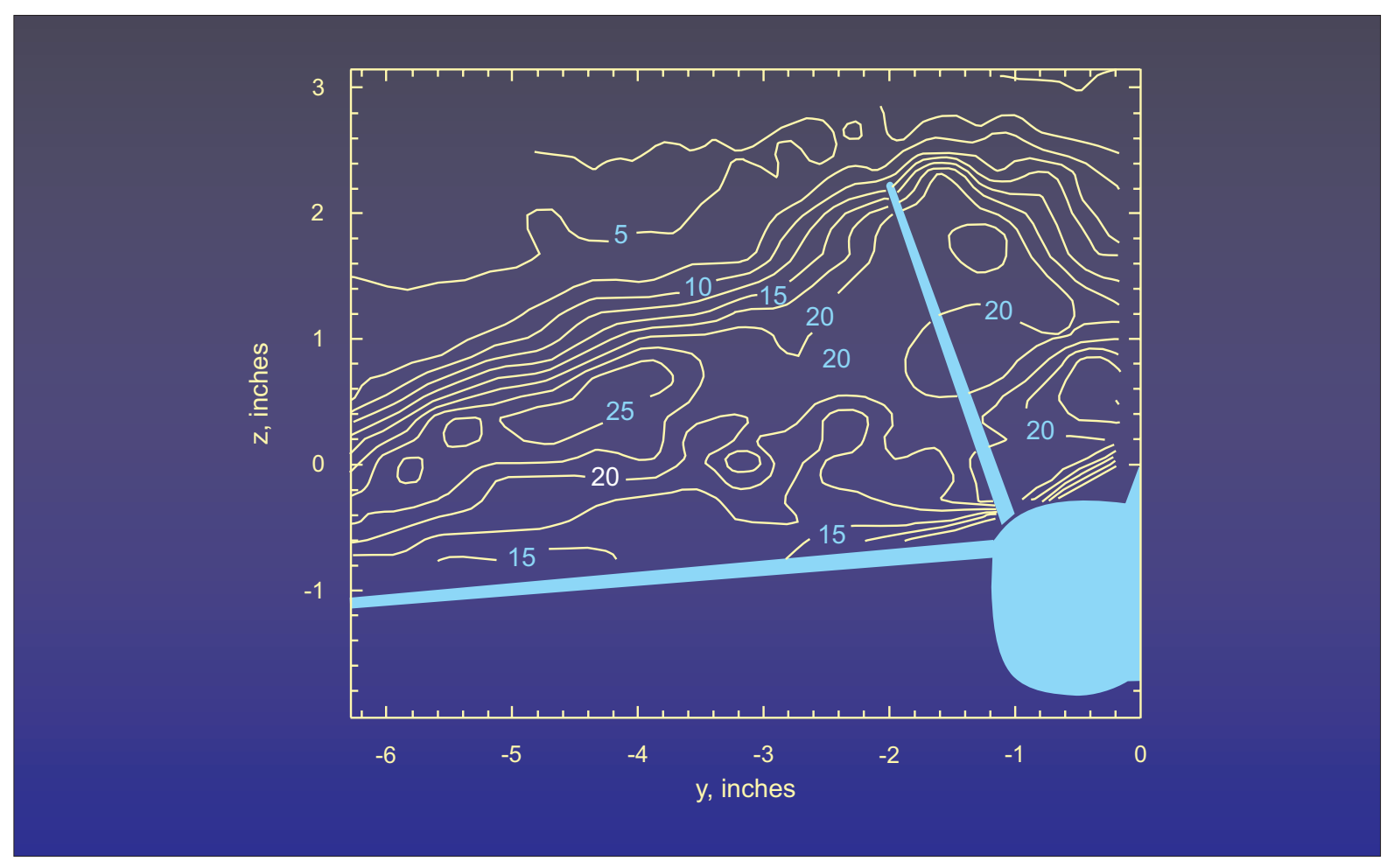

Figure 18c. RMS velocity contours, $\widetilde{w} / V_{\infty}$, percent, station 524; $\alpha=25^{\circ}, R_{n}=326,000$. 